\title{
Spectral measurement of the electron antineutrino oscillation amplitude and frequency using 500 live days of RENO data
}

\author{
S. H. Seo,${ }^{8}$ W. Q. Choi,${ }^{8}$ H. Seo, ${ }^{8}$ J. H. Choi, ${ }^{3}$ Y. Choi,${ }^{10}$ H. I. Jang, ${ }^{9}$ J. S. Jang, ${ }^{4}$ K. K. Joo, ${ }^{1}$ B. R. Kim, ${ }^{1}$
} H. S. Kim, ${ }^{7}$ J. Y. Kim, ${ }^{1}$ S. B. Kim, ${ }^{8}$ S. Y. Kim, ${ }^{8}$ W. Kim, ${ }^{6}$ E. Kwon, ${ }^{8}$ D. H. Lee, ${ }^{8}$ Y. C. Lee, ${ }^{8}$ I. T. Lim, ${ }^{2}$ M. Y. Pac, ${ }^{3}$ I. G. Park, ${ }^{5}$ J. S. Park,${ }^{8}$ R. G. Park, ${ }^{1}$ Y. G. Seon, ${ }^{6}$ C. D. Shin, ${ }^{1}$ J. H. Yang, ${ }^{10}$ J. Y. Yang, ${ }^{8}$ I. S. Yeo, ${ }^{1}$ and I. Yu ${ }^{10}$

\section{(RENO Collaboration)}

\author{
${ }^{1}$ Institute for Universe and Elementary Particles, Chonnam National University, Gwangju 61186, Korea \\ ${ }^{2}$ Department of Physics Education, Chonnam National University, Gwangju 61186, Korea \\ ${ }^{3}$ Department of Radiology, Dongshin University, Naju 58245, Korea \\ ${ }^{4}$ Department of Physics and Photon Science, Gwangju Institute of Science and Technology, \\ Gwangju 61005, Korea \\ ${ }^{5}$ Department of Physics, Gyeongsang National University, Jinju 52828, Korea \\ ${ }^{6}$ Department of Physics, Kyungpook National University, Daegu 41566, Korea \\ ${ }^{7}$ Department of Physics and Astronomy, Sejong University, Seoul 05006, Korea \\ ${ }^{8}$ Department of Physics and Astronomy, Seoul National University, Seoul 08826, Korea \\ ${ }^{9}$ Department of Fire Safety, Seoyeong University, Gwangju 61268, Korea \\ ${ }^{10}$ Department of Physics, Sungkyunkwan University, Suwon 16419, Korea
}

(Received 17 November 2016; published 3 July 2018)

\begin{abstract}
The Reactor Experiment for Neutrino Oscillation (RENO) has been taking electron antineutrino $\left(\bar{\nu}_{e}\right)$ data from the reactors in Yonggwang, Korea, using two identical detectors since August 2011. Using roughly 500 live days of data through January 2013 we observe 290775 (31 514) reactor $\bar{\nu}_{e}$ candidate events with $2.8 \%$ (4.9\%) background in the near (far) detector. The observed visible positron spectra from the reactor $\bar{\nu}_{e}$ events in both detectors show a discrepancy around $5 \mathrm{MeV}$ with regard to the prediction from the current reactor $\bar{\nu}_{e}$ model. Based on a far-to-near ratio measurement using the spectral and rate information, we have obtained $\sin ^{2} 2 \theta_{13}=0.082 \pm 0.009$ (stat.) \pm 0.006 (syst.) and $\left|\Delta m_{e e}^{2}\right|=\left[2.62_{-0.23}^{+0.21} \text { (stat. }\right)_{-0.13}^{+0.12}$ (syst.) $] \times 10^{-3} \mathrm{eV}^{2}$.
\end{abstract}

DOI: 10.1103/PhysRevD.98.012002

\section{INTRODUCTION}

The historical observations of neutrino oscillations [1-4] have verified that neutrinos are massive. The existence of neutrino mass requires modification of the Standard Model and provides hints on the grand unification theory. The smallest neutrino mixing angle $\theta_{13}$ in the Pontecorvo-Maki-Nakagawa-Sakata (PMNS) matrix $[5,6]$ was definitively measured in 2012 by Daya Bay [3] and RENO [4]. The leptonic $C P$ phase $\delta_{\mathrm{CP}}$ and neutrino mass ordering are now possible to be measured due to the large value of $\theta_{13}$. A precise measurement of $\theta_{13}$ by a reactor $\bar{\nu}_{e}$ experiment will greatly improve determination

Published by the American Physical Society under the terms of the Creative Commons Attribution 4.0 International license. Further distribution of this work must maintain attribution to the author(s) and the published article's title, journal citation, and DOI. Funded by SCOAP . of the $C P$ phase when combined with results of accelerator neutrino experiments $[7,8]$.

Using the two identical detectors in separate locations the RENO experiment measures the reactor $\bar{\nu}_{e}$ survival probability, $P_{e e} \equiv P\left(\bar{\nu}_{e} \rightarrow \bar{\nu}_{e}\right)[9]$,

$$
\begin{aligned}
P_{e e}= & 1-\sin ^{2} 2 \theta_{13}\left(\cos ^{2} \theta_{12} \sin ^{2} \Delta_{31}+\sin ^{2} \theta_{12} \sin ^{2} \Delta_{32}\right) \\
& -\cos ^{4} \theta_{13} \sin ^{2} 2 \theta_{12} \sin ^{2} \Delta_{21} \\
\approx & 1-\sin ^{2} 2 \theta_{13} \sin ^{2} \Delta_{e e}-\cos ^{4} \theta_{13} \sin ^{2} 2 \theta_{12} \sin ^{2} \Delta_{21},
\end{aligned}
$$

where $\Delta_{i j} \equiv 1.267 \Delta m_{i j}^{2} L / E_{\nu}, E_{\nu}$ is the $\bar{\nu}_{e}$ energy in $\mathrm{MeV}, L$ is the distance between the reactor and detector in meters, and $\Delta m_{e e}^{2}$ is the effective neutrino mass squared difference in $\mathrm{eV}^{2}$ and defined as $\Delta m_{e e}^{2} \equiv \cos ^{2} \theta_{12} \Delta m_{31}^{2}+$ $\sin ^{2} \theta_{12} \Delta m_{32}^{2}$ [10].

Recently RENO has published a letter [11] on the improved measurement of $\theta_{13}$ and the first measurement of $\left|\Delta m_{e e}^{2}\right|$ with a spectral shape and rate analysis using 
$\sim 500$ live days of data. This paper provides a more detailed description on the analysis.

\section{EXPERIMENTAL SETUP}

The RENO detectors are located near the Hanbit (previously known as Yonggwang) nuclear power plant, operated by Korea Hydro and Nuclear Power Co., Ltd. (KHNP), in Yonggwang, the southwest coast region in South Korea. The plant consists of six reactors linearly aligned with an equal distance of $\sim 260 \mathrm{~m}$ and provides a total thermal output of $16.8 \mathrm{GW}_{\text {th }}$. Reactors 1 and 2 each produce the maximum $2.755 \mathrm{GW}_{\text {th }}$ and newer reactors 3-6 each produce $2.755 \mathrm{GW}_{\text {th }}$.

RENO started its civil engineering in 2007 and completed the construction of two identical detectors, ready for data taking in June 2011. A near (far) detector is located at 294 (1383) $\mathrm{m}$ from the center of the six reactors. The near (far) detector is installed underground with an overburden of 120 (450) meter-water-equivalent (m.w.e). Figure 1 shows a layout of the RENO experiment.

The far-to-near ratio measurement using the two identical detectors greatly reduces the systematic uncertainties in the measurement of $\theta_{13}$ due to the cancellation of their correlated uncertainties. It would be difficult to measure the mixing angle $\theta_{13}$ with a single detector because of the large reactor $\bar{\nu}_{e}$ flux uncertainty. By measuring the reactor neutrino flux at the near detector and predicting the expected one at the far detector, the systematic error associated with the reactor $\bar{\nu}_{e}$ flux uncertainty can be significantly reduced. The baseline distances between the detectors and reactors are measured to an accuracy of better than $10 \mathrm{~cm}$ using GPS and total station.

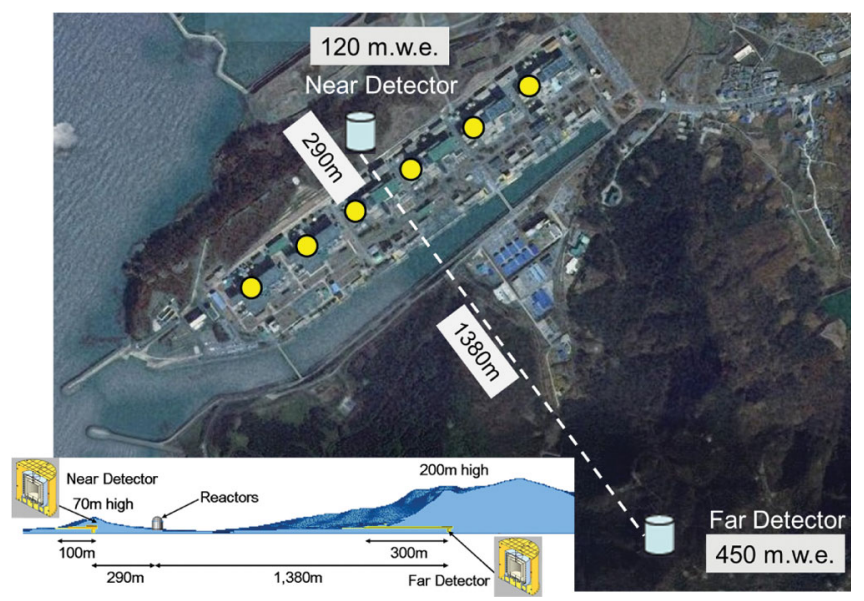

FIG. 1. (Top) Top view of the six reactors (circles) in Hanbit nuclear power plant and the location of the two detectors (cylinders). (Bottom) Side view of the RENO experimental layout.

\section{THE RENO DETECTOR}

The RENO experiment detects reactor $\bar{\nu}_{e}$ through the inverse beta decay (IBD) reaction, $\bar{\nu}_{e}+p \rightarrow e^{+}+n$, using a liquid scintillator (LS) with $0.1 \%$ gadolinium (Gd) as a target. In the IBD reaction $\bar{\nu}_{e}$ with energy larger than $1.81 \mathrm{MeV}$ interacts with a free proton in hydrocarbon LS to produce a positron and a neutron. The positron carries away the most kinetic energy of the incoming $\bar{\nu}_{e}$ while the neutron takes only $\sim 10 \mathrm{keV}$. The positron annihilates immediately to release $1.02 \mathrm{MeV}$ as two $\gamma$ rays in addition to its kinetic energy. The neutron after thermalization is captured by $\mathrm{Gd}$ with a mean delayed time of $\sim 26 \mu \mathrm{s}$.

RENO detectors are optimized to detect reactor $\bar{\nu}_{e}$ and consist of four layers of nested cylindrical structures as shown in Fig. 2. They are target, $\gamma$ catcher, buffer, and veto from the innermost and contain different liquids. The main inner detector (ID) is contained in a cylindrical stainless steel vessel of $5.4 \mathrm{~m}$ in diameter and $5.8 \mathrm{~m}$ in height and houses two nested cylindrical acrylic vessels. The $1.5 \mathrm{~m}$ thick outer detector (OD) surrounding the ID is filled with 350 tons of highly purified water. The OD is intended to identify events coming from the outside by their Cherenkov radiation and to shield against ambient $\gamma$ rays and neutrons from the surrounding rock.

The innermost target vessel, a $25 \mathrm{~mm}$ thick acrylic vessel of $2.75 \mathrm{~m}$ in diameter and $3.15 \mathrm{~m}$ in height, holds 16 tons of $0.1 \%$ Gd-doped LS (Gd-LS) as a neutrino target. It is surrounded by a $60 \mathrm{~cm}$ thick layer of 29 tons undoped LS in the $\gamma$ catcher, useful for recovering $\gamma$ rays escaping from

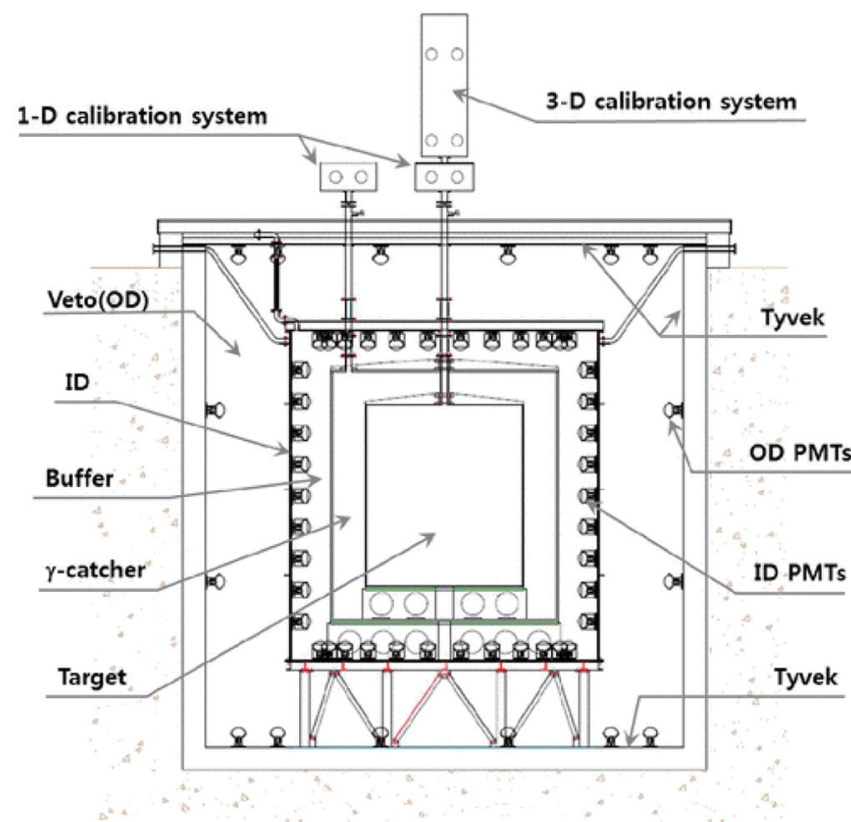

FIG. 2. The schematics of the RENO detector consisting of the ID (target, $\gamma$ catcher, and buffer) and OD (veto) detectors. A total of 354 (67) 10 in. PMTs detect scintillation lights from the ID (OD). 
the target region. The $\gamma$-catcher liquid is contained in a $30 \mathrm{~mm}$ thick acrylic vessel of $4.0 \mathrm{~m}$ in diameter and $4.4 \mathrm{~m}$ in height. The acrylic vessels holding organic liquids are made of casting polymethyl methacrylate [PMMA $\left(\mathrm{C}_{5} \mathrm{O}_{2} \mathrm{H}_{8}\right)_{n}$ ] plastic, which transmits up to $92 \%$ of visible light at $3 \mathrm{~mm}$ thickness and reflects about $4 \%$ from the surface in LS [12].

Outside the $\gamma$ catcher is a $70 \mathrm{~cm}$ thick buffer region filled with 65 tons of mineral oil. It provides shielding against ambient $\gamma$ rays and neutrons coming from outside. Light signals emitted from particles interacting in ID are detected by 354 low-background 10 in. Hamamatsu R7081 photomultiplier tubes (PMTs) [13] that are mounted on the inner wall of the ID. The OD is equipped with $6710 \mathrm{in.} \mathrm{R7081}$ water-proof PMTs mounted on the wall of the concrete veto vessel. The inner surface of OD is covered with Tyvek sheets to increase the light collection.

The LS is developed and produced as a mixture of linear alkylbenzene (LAB), $3 \mathrm{~g} / \ell$ of 2,5-diphenyloxazole (PPO), and $30 \mathrm{mg} / \ell$ of p-bis-(o-methylstyryl)-benzene (bisMSB). LAB $\left(\mathrm{C}_{n} \mathrm{H}_{2 n+1}-\mathrm{C}_{6} \mathrm{H}_{5}, n=10-13\right)$ is an organic solvent with a high flash point of $130^{\circ} \mathrm{C}$ and has a good light yield and a large attenuation length. A Gd-carboxylate complex using trimethylhexanoic acid was developed for the best Gd loading efficiency into LS and its long term stability [14]. Care is taken in the production of LS and Gd-LS and filling into detectors to ensure that the near and far detectors are as identical as possible.

The RENO detector uses Cartesian coordinates of $x, y$, and $z$ with an origin at the center of the detector. The $z$ coordinate is along the cylindrical axis. The detectors are calibrated using radioactive sources and cosmic-ray induced backgrounds. Various radioisotopes of gammaray sources are periodically deployed in the target and gamma-catcher by a motorized pulley system in a glove box as shown in Fig. 2. The system deploys a source along the vertical direction only. The relative source location can be controlled at an accuracy of a few millimeters by a stepper motor, but absolute vertex location accuracy is $1 \mathrm{~cm}$. The source data are taken every one or two months to monitor the detector stability and to obtain calibration parameters. Also a three-dimensional (3D) calibration system is developed for deploying calibration sources at off cylindrical axis positions in the target. However, it has not been used. More details on the RENO detector are found in Ref. [15].

\section{DATA ACQUISITION}

The scintillation light produced in the liquid scintillator from the interaction of signal or background events is collected by the PMTs. Analog signals are produced and sent through $25 \mathrm{~m} \mathrm{RG303/U}$ single cables to the signal processing front-end boards. The RENO data acquisition (DAQ) system uses electronic modules developed for the Super-Kamiokande experiment [16] and consists of a total of 18 front-end boards with 24 channels each, driven by a common $60 \mathrm{MHz}$ master clock. Each front-end board is equipped with eight charge-to-time conversion (QTC) chips, four time-to-digital converter (TDC) chips, and a 100 Mbps ethernet card. The QTC chip has three inputs with different gains of 1,7 , and 49 to cover a dynamic range from 0.2 to $2500 \mathrm{pC}$ with a resolution of $0.1 \mathrm{pC}$ at gain 1. The QTC chip measures the time and integrated charge of a PMT analog signal and converts them to digital values. The timing information is fed into a TDC chip to be digitized and recorded. The signal processing time per hit is roughly $800 \mathrm{~ns}$ for charge sampling and digitization. The signal front-end boards can handle up to $\sim 100 \mathrm{kHz}$ of events each with photon hits on every PMT without dead time and does not require any hardware triggers to lower the event rate.

An off-line software trigger system generates buffer, veto, or buffer and veto triggers for an event if it satisfies an appropriate trigger condition. The number of PMT hits ( $N_{\text {hit }}$ is defined as the number of PMTs that has a signal larger than 0.3 p.e. in a $50 \mathrm{~ns}$ time window. A buffer trigger requires ID $N_{\text {hit }}>90$, corresponding to $0.5-0.6 \mathrm{MeV}$, and is well below the $1.02 \mathrm{MeV}$ minimum energy of an IBD positron signal. Upon a trigger an event is made by collecting all the PMT hits in a time window of -18 to $+18 \mu \mathrm{s}$. The time zero is defined by the first hit time when $N_{\text {hit }}$ is greater than 90 in a time window of $50 \mathrm{~ns}$. The only PMT hits in a time window of -100 to +50 ns are used for the event energy and vertex reconstruction. The PMT hits outside a time window of -100 to +50 ns are recorded to monitor dark currents. If a trigger is issued within $18 \mu \mathrm{s}$ of the previous trigger, the PMT hits in the overlapping time windows are shared between two events. A veto trigger is issued for a cosmic-ray muon event and requires OD $N_{\text {hit }}>10$ out of a total of 67 OD PMTs. A buffer and veto trigger is issued if an event satisfies the two conditions simultaneously.

The average total trigger rates of the 500 day data sample are $\sim 590 \mathrm{~Hz}$ in the near detector and $\sim 140 \mathrm{~Hz}$ in the far detector. The trigger types and rates are summarized in Table I. The buffer-only trigger is required for an IBD candidate, and the rate is $\sim 60(\sim 77) \mathrm{Hz}$ for the near (far) detector. The veto-only trigger rate is higher in the near detector having less overburden than the far detector.

TABLE I. Average trigger rates of the $~ 500$ live days of data in the RENO detectors. The rates for the buffer-only trigger required for the IBD event are $\sim 60 \mathrm{~Hz}$ (near) and $\sim 77 \mathrm{~Hz}$ (far).

\begin{tabular}{lcc}
\hline \hline Trigger type & Near [Hz] & Far [Hz] \\
\hline Buffer & 269 & 100 \\
Veto & 529 & 61 \\
Buffer and veto & 209 & 23 \\
Total & 590 & 138 \\
\hline \hline
\end{tabular}


TABLE II. Reactor-off periods during the $\sim 500$ live days.

\begin{tabular}{lc}
\hline \hline Periods & Off reactor number \\
\hline $2011.08 .30-2011.09 .29$ & 2 \\
$2012.02 .24-2012.03 .21$ & 1 \\
$2012.05 .01-2012.05 .30$ & 5 \\
$2012.06 .07-2012.07 .17$ & 4 \\
$2012.10 .19-2012.11 .07$ & 4 \\
$2012.11 .08-2012.12 .30$ & $3,4,5$ \\
$2012.12 .31-2013.01 .21$ & 3 \\
\hline \hline
\end{tabular}

Real-time online monitoring for PMT hit rates, trigger rates, high voltage $(\mathrm{HV})$, and other interesting variables is performed to find possible data-taking troubles. Various environmental parameters including the water level and temperature are also monitored online [17]. More checks are performed off-line on a weekly basis for trigger rates, muon rates, flashing PMT rates, IBD prompt and delayed candidate rates, and charge stability.

\section{DATA SAMPLE}

RENO has started taking data in August 2011 and has been operating continuously so far with an accumulated average DAQ efficiency of better than $95 \%$ for both detectors. In this analysis 489.93 (458.49) live day data with negligible uncertainties in the far (near) detector taken from August 2011 to January 2013 are used to extract the neutrino mixing parameters, $\theta_{13}$ and $\left|\Delta m_{e e}^{2}\right|$. Each reactor is periodically turned off for a month every 1.5 years to replenish nuclear fuel. Besides these periodic turn-offs there are sporadic unscheduled down times. All of this information is provided by KHNP. Table II summarizes the reactor-off periods during the $\sim 500$ live days.

\section{DETECTOR SIMULATION}

The primary software tool for modeling the RENO detector response is GLG4SIM [18], a GEANT4 ${ }^{1}$ based simulation package for liquid scintillator detectors derived from KLG4SIM of the KamLAND Collaboration. The GLG4SIM is designed for simulation of the detailed detector response to particles moving through and interacting with a large volume of liquid scintillator detector. This generic program has been customized for the RENO detector. The GEANT4 toolkits are used for simulating the physics processes involving particles with energies above a few $\mathrm{keV}$ propagating through the detector materials. However, the optical photon production and propagation through liquid scintillator, including processes such as absorption, reemission, and elastic collisions, are handled by specifically written codes in GLG4SIM, using measured optical properties of the RENO LS. The simulation includes the

\footnotetext{
${ }^{1}$ V4.7.1 patch01.
}

measured quenching effect of the $\gamma$ ray at low energies using a pure Ge detector.

GLG4SIM has a detailed modeling of PMTs and takes into account transmission, absorption, and reflection of optical photons at the photocathode. The photocathode thickness and wavelength dependent photocathode efficiency are implemented in the PMT model.

Each photon generated in the simulation is tracked in the detector until it either reaches a PMT or is lost. The simulation takes into account several light propagation phenomena while tracking the photons. In the scintillator, photons can be absorbed or elastically scattered (Rayleigh scattering) by solvent and fluor molecules.

The absorption of photons within the acrylic vessel medium is simulated according to the absorption probability calculated with the medium's attenuation length. Also, the reflection and refraction of photons at the surface of the acrylic vessel are simulated using Fresnel's law. The refractive indices of all dielectric materials in the detector are measured at different wavelengths and implemented in the simulation.

For the simulation of neutron capture on $\mathrm{Gd}$, the GLG4SIM is used to provide a proper modeling of discrete lines of high-energy gammas and the continuous gamma spectrum arising from the neutron capture on Gd. Both Cherenkov radiation and scintillation light emission are simulated.

The GEANT used in the Monte Carlo (MC) simulation is outdated due to time evolution since the start of RENO and therefore needs to be updated. However, this measurement is largely data driven and thus expects to be hardly affected by the update. Systematic uncertainties may be improved by better understanding the detailed physics processes with an updated MC simulation.

The dead PMT fraction during the data taking reported here is less than $1 \%$ for both near and far detectors. However, the dead PMTs are not accounted for in RENO MC simulation as the time dependent charge correction in data compensates for the effects of dead PMTs. More details on RENO detector simulation is found in [15].

\section{EVENT RECONSTRUCTION}

Reconstructed energy and vertex are essential for selecting IBD candidate events against various backgrounds. In the following subsections we describe energy and vertex reconstructions of the triggered events.

\section{A. Energy reconstruction}

An analog signal from each PMT is amplified, integrated, and then digitized by analog-to-digital converter (ADC) in a QTC chip. The ADC value is then converted to a charge in pC. A charge injection board is used to determine an ADC-to-pC conversion factor for an individual channel of a front-end board. Using a ${ }^{137} \mathrm{Cs}$ source, a fit to one photoelectron response finds a corresponding charge 


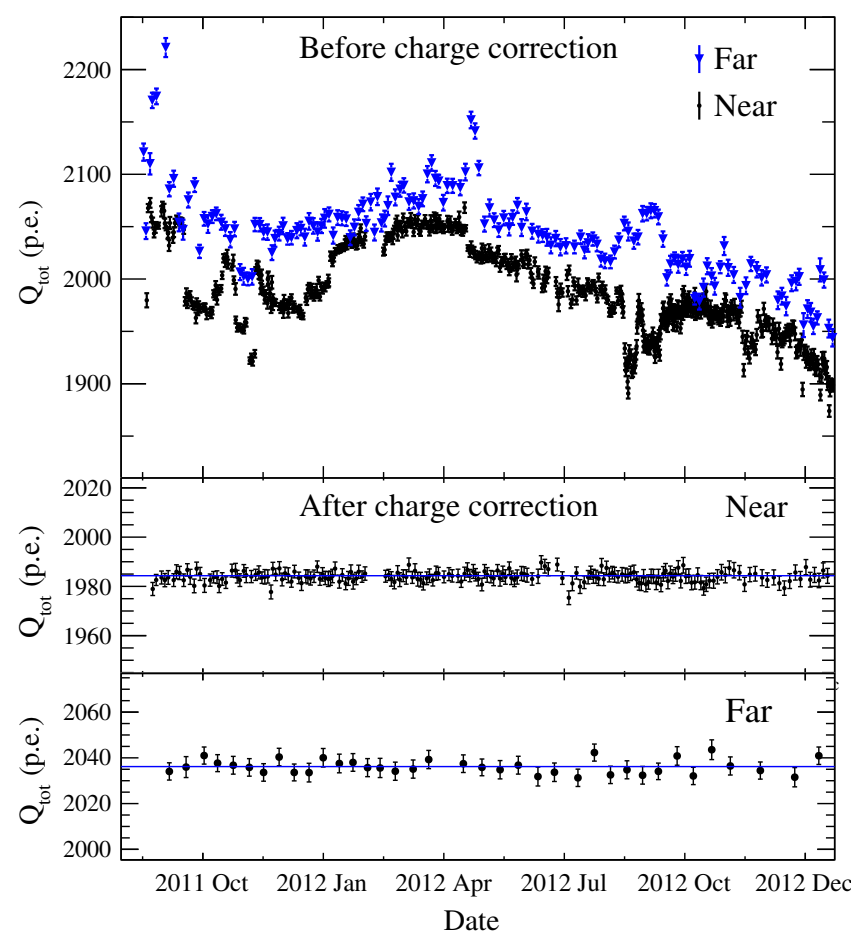

FIG. 3. Time variation of raw charges observed by IBD delayed events, i.e., $n$-Gd capture event peaks (top panel), and time stability of their corrected charges (bottom panels).

value of $\sim 1.6 \mathrm{pC}$. A PMT charge is measured in p.e. based on the conversion factor.

The event energy is determined by the total charge $\left(Q_{\text {tot }}\right)$ that is defined as a sum of hit PMT charges greater than 0.3 p.e. in a time window of -100 to +50 ns. The event time window is determined by taking into account the size of the RENO detector and minimizing the contributions of dark hits, flashing PMT hits, and negative charges caused by the unsettled pedestal after a large pulse height due to a highly energetic muon.

The raw $Q_{\text {tot }}$ of IBD delayed signals shows a time variation as shown in the upper panel of Fig. 3. This is caused by PMT gain change, removal of flashing PMTs, and the decrease of the LS attenuation length [19]. The raw charge time variation is corrected using temporal charge correction factors obtained from the IBD delayed signal peaks with respect to a reference value. The lower panel of Fig. 3 shows an excellent stability of the reconstructed energies of IBD delayed signals after the temporal charge correction. According to the charge uniformity map shown in Fig. 4, there is no need of a spatial charge correction since the charge differences of less than $1 \%$ in the entire target volume are observed. The nonuniform energy response near the target acrylic vessel is due to the loss of energy in the acrylic and due to larger scintillation of spill-in events in the $\gamma$ catcher. The energy loss effect is a bit pronounced at the bottom due to the acrylic structure supporting the target and $\gamma$-catcher vessels. This energy
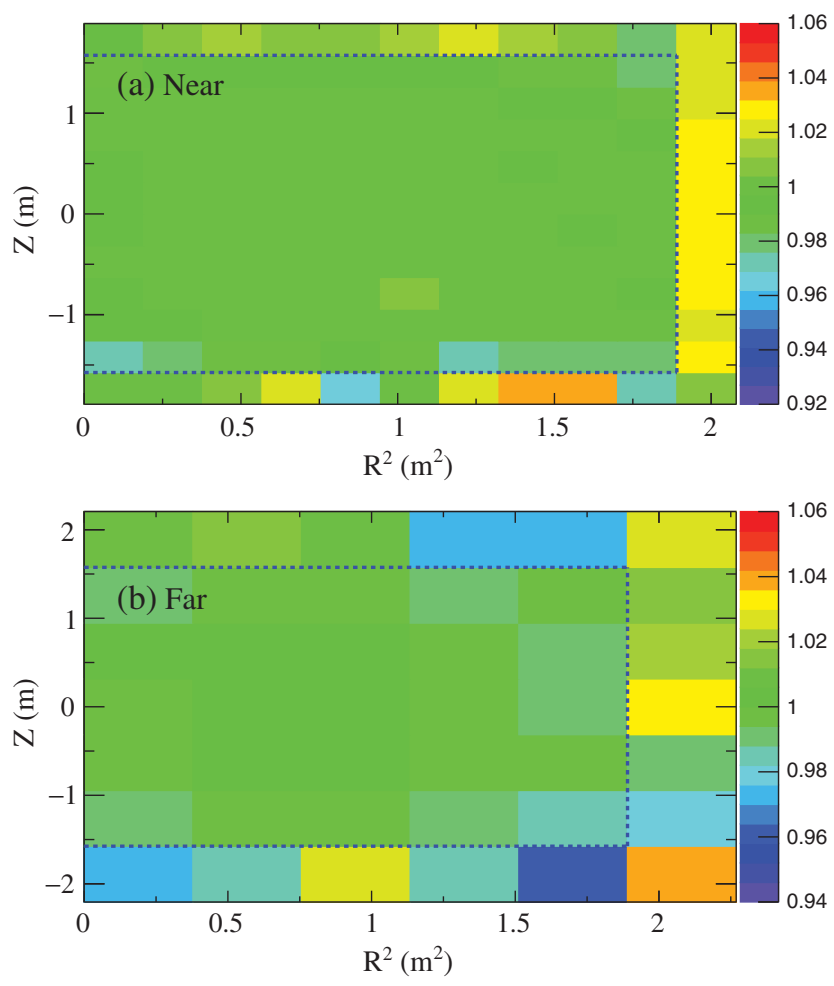

FIG. 4. Charge uniformity map seen with $n$-Gd delayed energy peaks in the (a) near and (b) far detectors. The radial coordinate $R$ is defined by $\sqrt{x^{2}+y^{2}}$. The blue dotted line is a target boundary. The color code represents the ratio of the fitted corrected charges in a bin to a reference value.

loss introduces a slight modification of the energy spectrum of prompt events in a few percent $(<4 \%)$ level, but occurs identically in the near and far detectors. Again our far-tonear ratio measurement minimizes a possible spectral difference between the two detectors.

After the raw charge correction of $Q_{\text {tot }}$ described above, $Q_{\text {tot }}$ in p.e. is converted to energy in $\mathrm{MeV}$ using an energy conversion function that will be described in the calibration section later. After the charge correction and conversion we obtain reconstructed energies. Figure 5 shows a good agreement between data and MC simulation in the delayed signal spectrum of IBD candidate events.

\section{B. Muon energy estimation}

Cosmogenic muons introduce a main background in the IBD candidates. The intrinsic muon energy cannot be reconstructed, but its deposited energy inside the detector can reasonably be measured as a visible energy proportional to its path length. The muon deposit energy $\left(E_{\mu}\right)$ is reconstructed by the observed $Q_{\text {tot }}$ with a conversion factor of 250 p.e. per MeV. A muon is identified by an event with the deposit energy greater than $70 \mathrm{MeV}$. Because of the saturation of the DAQ electronics the muon deposit energy cannot exceed a maximum value $\sim 1700 \mathrm{MeV}$ as shown in Fig. 6. The muon charge correction is obtained from the 


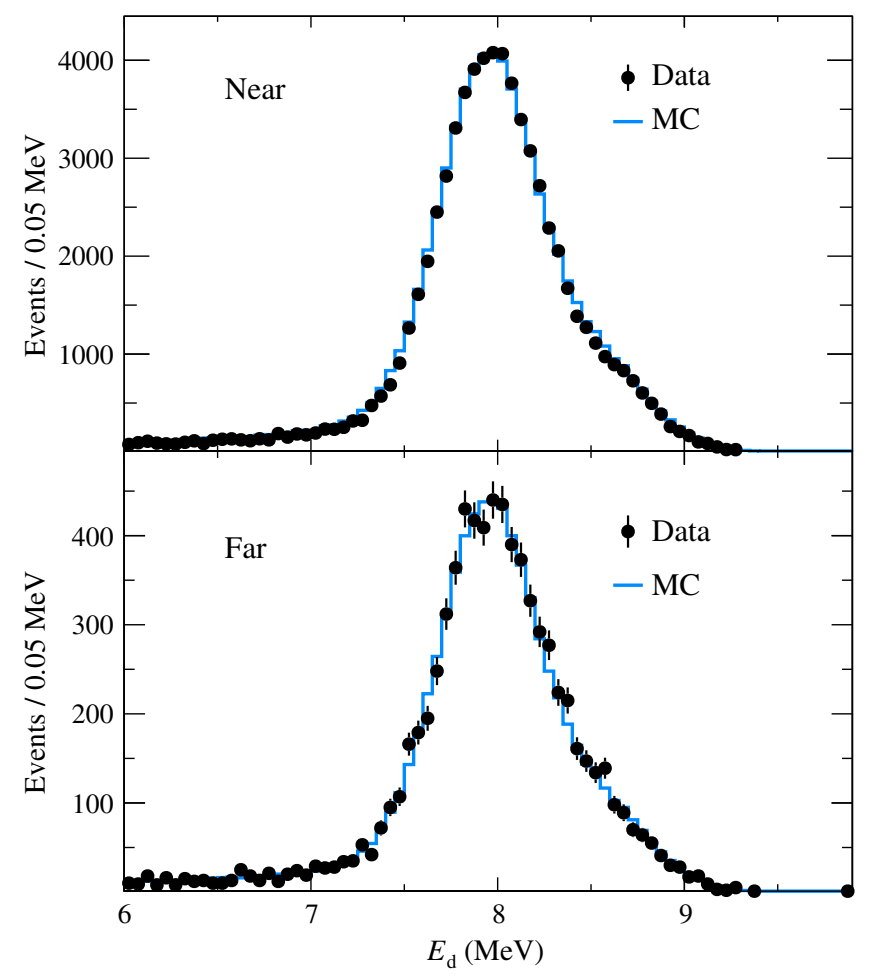

FIG. 5. Delayed energy spectra of the IBD candidate events in the near and far detectors. Data and MC simulation spectra agree well.

change of the maximum deposit energy with respect to a reference value.

\section{Vertex reconstruction}

The event vertex information is useful for removing accidental backgrounds because of their uncorrelated distances between prompt and delayed candidates. A simple and fast method is adopted to reconstruct an event vertex using an individual PMT charge as a weighting factor to the position of a hit PMT. A reconstructed vertex, $\vec{r}_{\mathrm{vtx}}$, is

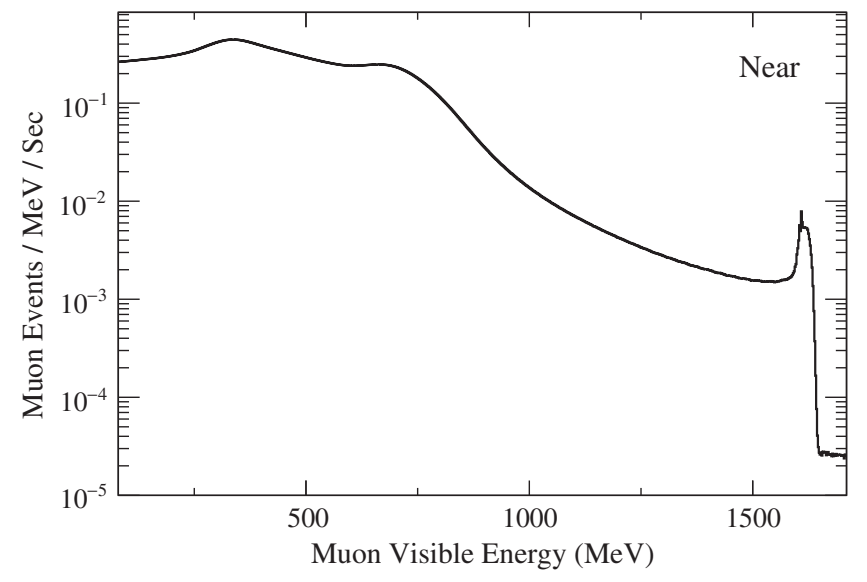

FIG. 6. Muon deposit energy distribution for the near detector. The maximum energy of $\sim 1700 \mathrm{MeV}$ is due to the saturation of the DAQ electronics.

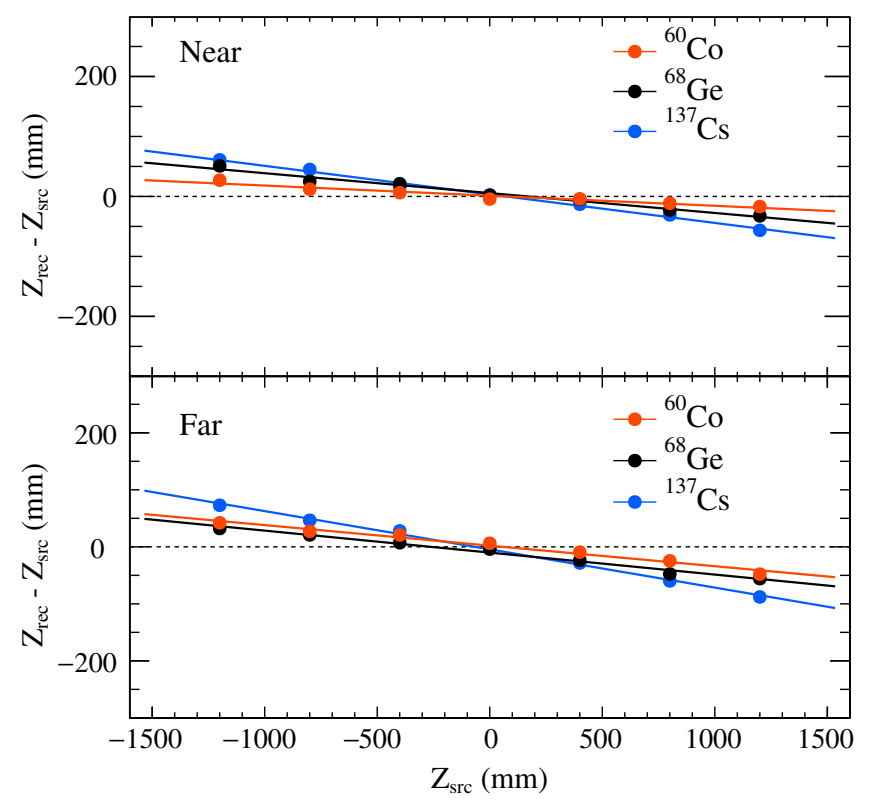

FIG. 7. Difference between reconstructed vertices $\left(Z_{\text {rec }}\right)$ and actual positions $\left(Z_{\text {src }}\right)$ of ${ }^{137} \mathrm{Cs},{ }^{68} \mathrm{Ge}$, and ${ }^{60} \mathrm{Co}$ sources. The reconstructed vertices show systematic deviations from the true positions at the source locations away from the center. The systematic shifts become lessened as the source energy is larger.

obtained as a charge weighted average of locations of all the hit PMTs,

$$
\vec{r}_{\mathrm{vtx}}=\frac{\sum_{i}\left(Q_{i} \cdot \vec{r}_{i}\right)}{\sum_{i} Q_{i}}
$$

where $Q_{i}$ is the charge collected by the $i$ th PMT, and $\vec{r}_{i}$ is a position vector of the PMT from the center of the RENO detector [20]. This method results in $\vec{r}_{\mathrm{vtx}}$ with a position dependent offset from the true vertex position mainly due to geometrical effects. A correction factor that depends on $\vec{r}_{\mathrm{vtx}}$ is obtained using simple numerical calculations that account for a simple geometrical shape of detector and the effective attenuation length of ID materials.

The performance of the vertex reconstruction was checked with three calibration source data: ${ }^{137} \mathrm{Cs},{ }^{68} \mathrm{Ge}$, and ${ }^{60} \mathrm{Co}$. The vertex resolution is about $20 \mathrm{~cm}$ at $1 \mathrm{MeV}$, and improves at higher energies. Figure 7 shows a reasonable agreement between the reconstructed and the actual source positions. The difference is as large as $\sim 7 \%$ for ${ }^{137} \mathrm{Cs}$ and less than $\sim 5 \%$ for the other two sources with gamma-ray energies larger than $1 \mathrm{MeV}$. However, such a bias is not really problematic because the requirement of a delayed signal from neutron capture on Gd naturally selects the target events without the event vertex information.

\section{ENERGY CALIBRATION}

An energy measurement is essential for measuring $\left|\Delta m_{e e}^{2}\right|$ and $\theta_{13}$ to a lesser extent. To calibrate the energy scale we used the following radioactive sources with a 
$\mu \mathrm{Ci}$ level or lower activities: ${ }^{137} \mathrm{Cs},{ }^{68} \mathrm{Ge},{ }^{60} \mathrm{Co},{ }^{210} \mathrm{Po}{ }^{9} \mathrm{Be}$, and ${ }^{252} \mathrm{Cf}$. The source is enclosed in an acrylic container when taking the source data. Source data are taken regularly, and their observed charges are corrected for variations of gain, charge collection, and LS attenuation length using the neutron capture peak energies. The corrected charges are averaged and used to represent $Q_{\text {tot }}$ for the peak energy of a $\gamma$-ray source. The total charge $Q_{\text {tot }}$, given in p.e., is converted to the corresponding absolute energy in $\mathrm{MeV}$ causing a charge-to-energy conversion function obtained through various source calibration samples and neutron capture samples. The conversion function from $Q_{\text {tot }}$ to corresponding energy deposited by a positron is generated from the peak energies of these $\gamma$-ray sources.

The observed charges of the source data, taken at the detector center, are also corrected for a different charge response of uniformly distributed events. The center-touniform corrections are $\sim 0.7 \%$ and $\sim 0.5 \%$ for the near and far detectors. The energy loss due to the source wrapper and container is estimated with MC simulation calculation and accounted for accordingly. The RENO MC simulation includes measured optical properties of the LS and the quenching effect of the $\gamma$ ray at low energies [14]. The quenching effect depends on the energy and the multiplicity of $\gamma$ ray released from the calibration sources. The MC simulated $Q_{\text {tot }}$ well reproduces that of the $\gamma$-ray source including the quenching effect.

Since a positron loses its kinetic energy via scintillating processes and annihilates with an electron and emitting two $\gamma$ rays, its total energy is taken as the true energy $\left(E_{\text {true }}\right)$ of the positron. The observed $Q_{\text {tot }}$ of the $\gamma$-ray source is converted to the corresponding Qtot of a positron $\left(Q_{\mathrm{tot}}\right)$ after all the necessary corrections using the GEANT4. The $Q_{\text {tot }}$ correction from $\gamma$ ray to positron is performed by taking the $\gamma$-ray source energy as the positron $E_{\text {true }}$ or corresponding IBD prompt energy $\left(E_{\mathrm{p}}\right)$. The converted $Q_{\mathrm{tot}}^{c}$ of IBD prompt energy is estimated by taking into account the difference in the visible energies of the $\gamma$ ray and positron through the MC simulation. The uncertainty in $Q_{\text {tot }}^{c}$ due to the correction is largely correlated among data points and negligible compared to the source data errors including the time variation of corrected charges. The upper panels of Fig. 8 show the nonlinear response of scintillating energy for the IBD prompt signal that is well described by a fitted parametrization and consistent with the MC prediction. The nonlinear response at lower energies is mainly due to the quenching effect in the scintillator and Cherenkov radiation. The following empirical formula is used for the fit function:

$$
Q_{\text {tot }}^{c} / E_{\text {true }}=P_{0}-P_{1} /\left[1-\exp \left(-P_{2} \cdot E_{\text {true }}-P_{3}\right)\right],
$$

where $E_{\text {true }}$ is in $\mathrm{MeV}$. The fit parameters $P_{0}$ determine a saturation level $P_{1}$ corresponding to the magnitude of
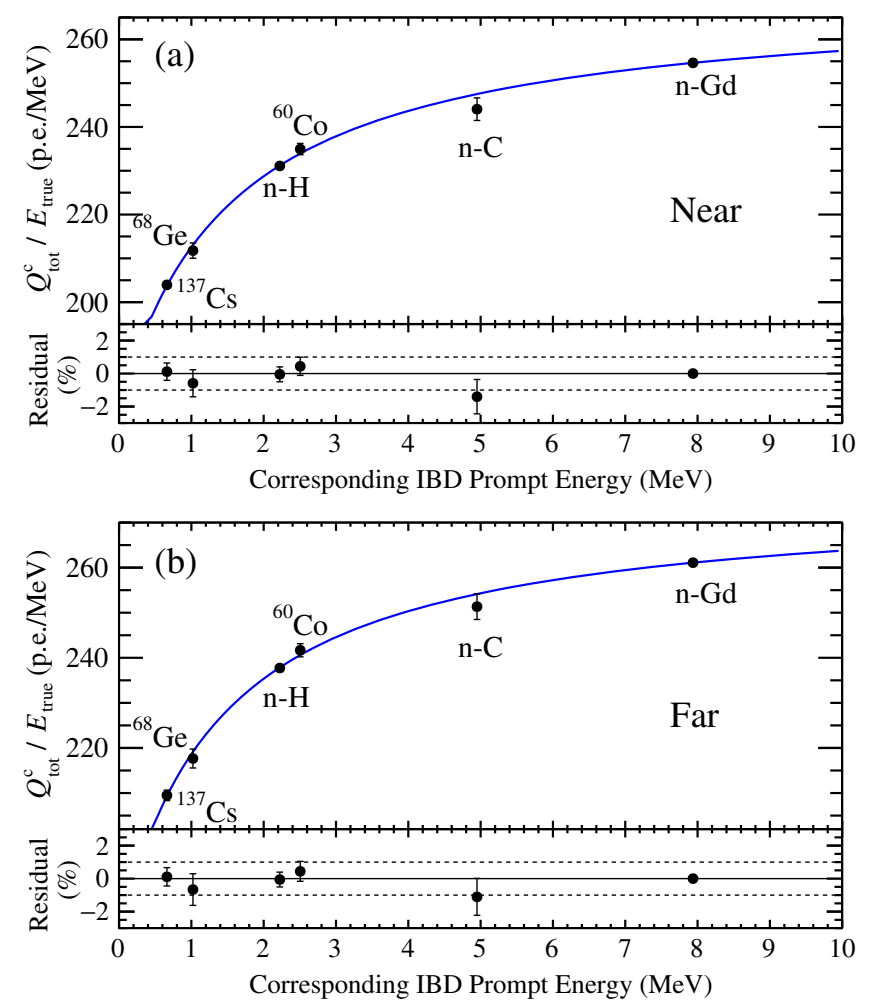

FIG. 8. Nonlinear response of scintillating energy obtained from the visible energies of $\gamma$ rays coming from several radioactive sources and IBD delayed signals in the near and far detectors. The curves are the best fits to the data points and the charge-to-energy conversion functions. Note that the $n$ - $\mathrm{C}$ sample is obtained from the ${ }^{210} \mathrm{Po}^{9} \mathrm{Be}$ source and the $n-\mathrm{H}$ sample from the ${ }^{252} \mathrm{Cf}$ source. The lower panels show fractional residuals of all calibration data points from the best fit.

nonlinearity, and $P_{2}$ and $P_{3}$ are related to the shape of the nonlinearity. The fitted values of the parameters are presented in Table III. The deviation of all calibration data points with respect to the best fit is within $1 \%$ as shown in Fig. 8 lower panels. According to the energy calibration, the observed charge $Q_{\text {tot }}$ at the far detector is $\sim 220$ p.e. per $\mathrm{MeV}$ at $1 \mathrm{MeV}$, and $\sim 250$ p.e. per $\mathrm{MeV}$ at $5 \mathrm{MeV}$.

The effective attenuation lengths of the near and far detectors differ by $1.4 \%$ at $430 \mathrm{~nm}$ wavelength that is estimated by PMT charge response to the radioactive source at the detector center. The LS light yields of the two detectors differ by $2.7 \%$ at $\sim 1 \mathrm{MeV}$. The dead PMT

TABLE III. The fit parameter values of the charge-to-energy conversion function given in Eq. (3).

\begin{tabular}{lcc}
\hline \hline Parameter & Far & Near \\
\hline$P_{0}$ & $275.9 \pm 1.0$ & $270.1 \pm 1.3$ \\
$P_{1}$ & $(1.698 \pm 0.151) \times 10^{-2}$ & $(1.701 \pm 0.247) \times 10^{-2}$ \\
$P_{2}$ & $(1.228 \pm 0.123) \times 10^{-4}$ & $(1.161 \pm 0.117) \times 10^{-4}$ \\
$P_{3}$ & $(1.735 \pm 0.176) \times 10^{-4}$ & $(1.794 \pm 0.299) \times 10^{-4}$ \\
\hline \hline
\end{tabular}




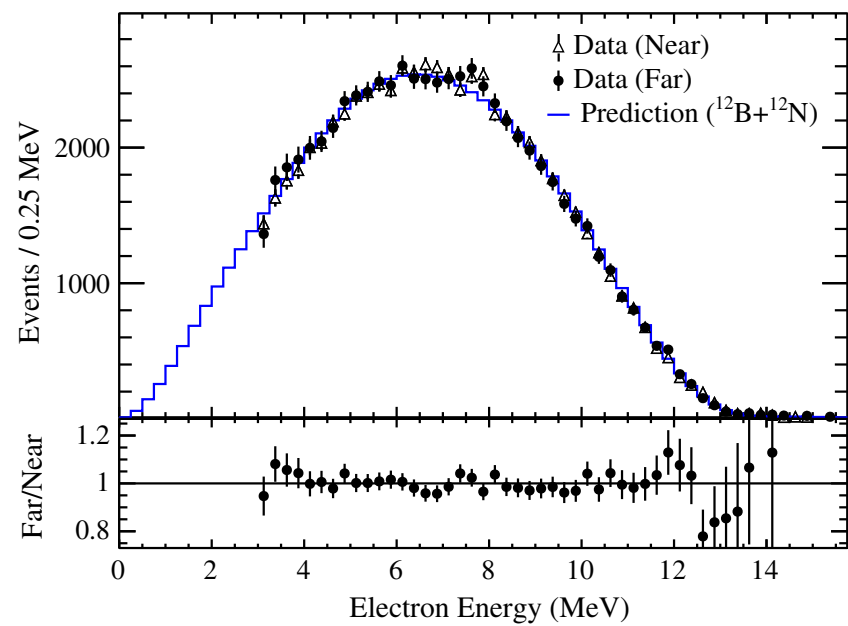

FIG. 9. Comparison of measured and simulated energy spectra of the electrons from $\beta$ decay of unstable isotope ${ }^{12} \mathrm{~B}$, with minute contribution from ${ }^{12} \mathrm{~N}$, produced by cosmic muons. The spectra are overlaid after scaling the total number of events in the near detector to that in the far detector. The far-to-near ratio of the spectra is shown in the lower panel. A small excess near $8 \mathrm{MeV}$ is seen in both near and far detectors and may be remaining background events coming from neutron capture by Gd.

fraction during the data taking reported here is less than $1 \%$ for both near and far detectors, and the difference between them is less than $0.5 \%$. This difference is compensated when the charge-to-energy conversion is performed using the conversion function obtained for each detector.

Cosmogenic ${ }^{12} \mathrm{~B}$ and ${ }^{12} \mathrm{~N}$ samples are used to check the validity of the charge-to-energy conversion functions. These isotopes are generated by cosmic muons interacting with carbons in the scintillator. The positron charge-to-energy conversion functions are modified to convert the charge in the $\beta$-decay events by subtracting a charge value corresponding to the positron annihilation. Figure 9 shows good agreements in the energy distributions between near and far data as well as data and MC simulation. This demonstrates the obtained parametrization for the nonlinear response of electron scintillating energy works well for energies of 3 to $14 \mathrm{MeV}$ within the statistical fluctuation of the data sample. Thus it indicates the positron energy conversion function is valid not only for the IBD energy region up to $8 \mathrm{MeV}$ but also for the extended energy region up to $14 \mathrm{MeV}$.

The energy-scale difference between the near and far detectors contributes to the uncorrelated systematic uncertainties associated with a relative measurement of spectra at two detectors, whereas the correlated uncertainties to the absolute energy scale does not. The energy-scale difference is estimated by comparing near and far spectra of calibration data and is found to be less than $0.15 \%$ as shown in Fig. 10.

The energy resolution is measured with the calibration data with the radioactive sources placed at the center of the detector. The obtained energy resolution is $\sigma / E=7.9 \% / \sqrt{E(\mathrm{MeV})+0.3}$ for the far detector with a

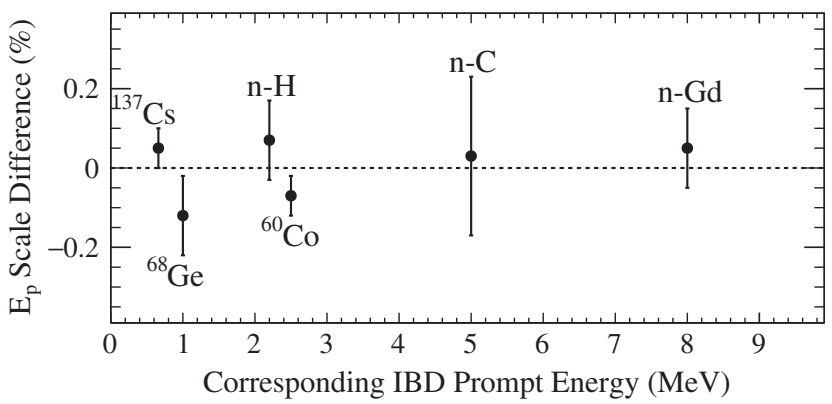

FIG. 10. Energy-scale difference between the near and far detectors. The prompt energy difference between the two detectors is estimated by comparing the energy spectra of $\gamma$-ray sources obtained using the charge-to-energy conversion functions. All calibration data show the values of the difference less than $0.15 \%$.

comparable energy resolution for the near detector. The discrepancy between data and MC simulation is taken into account in MC simulation. The energy resolution is worse by a small amount due to IBD events being uniformly distributed in the target region. The difference is estimated to be less than $0.2 \%$. The dotted curve is the energy resolution used for the results in Ref. [11]. An updated resolution is obtained to be more appropriate for the uniform IBD events. The difference between the two energy resolution functions is minimal as shown by their residual distribution in the lower panel of Fig. 11. The measurement of $\sin ^{2} 2 \theta_{13}$ and $\left|\Delta m_{e e}^{2}\right|$ is repeated with the updated energy resolution, and the obtained values are essentially unchanged except for an increase of $0.01 \times 10^{-3} \mathrm{eV}^{2}$ in the $\left|\Delta m_{e e}^{2}\right|$ value. Therefore, the energy resolution function used in Ref. [11] is taken for the results in this paper.

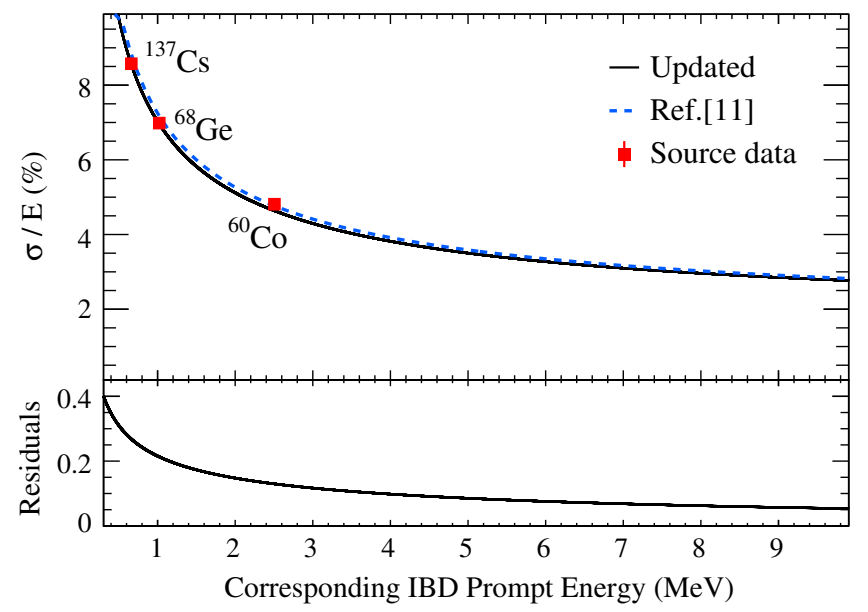

FIG. 11. Energy resolution for the far detector as a function of prompt energy. The solid curve corresponds to the estimated energy resolution using a MC simulation. The dotted curve represents the energy resolution used in Ref. [11]. Their difference is shown as a residual in the lower panel. Note that each source data point is given at the true energy of a gamma ray(s). 


\section{BACKGROUNDS}

There are several background contributions to the prompt- and delayed-like events. They are ambient $\gamma$ rays from the surrounding rock and the detector materials, neutrons entering into the detector, spallation products produced by the cosmic muons, flashing lights from PMTs, electronic noise, and others. Two main background components for the IBD candidates are uncorrelated and correlated pairs of prompt- and delayed-like events. Because of a much shallower overburden for the near detector than the far detector, the near detector suffers a higher rate of cosmogenic backgrounds.

The uncorrelated IBD background is due to accidental coincidences from the random association of a prompt-like event due to radioactivity and a delayed-like neutron capture. The prompt-like events are mostly ambient $\gamma$ rays from the radioactivity in the PMT glasses, LS, and surrounding rock. Most of the ambient radioactivities generate $\gamma$ rays of energies below $3 \mathrm{MeV}$. The delayedlike events come from neutrons produced by cosmic muons in the surrounding rocks or in the detector.

The correlated IBD backgrounds are due to fast neutrons, $\beta$ - $n$ emitters from cosmogenic ${ }^{9} \mathrm{Li} /{ }^{8} \mathrm{He}$ isotopes, and ${ }^{252} \mathrm{Cf}$ contamination in the target. The fast neutrons are produced by cosmic muons traversing the surrounding rock and the detector. An energetic neutron entering the ID can interact in the LS to produce a recoil proton before being captured on $\mathrm{Gd}$. The recoil proton generates scintillation lights mimicking a prompt-like event. The ${ }^{9} \mathrm{Li} /{ }^{8} \mathrm{He} \beta-n$ emitters are produced mostly by energetic cosmic muons because their production cross sections in carbon increase with muon energy.

The ${ }^{252} \mathrm{Cf}$ contamination background comes from the contamination of Gd-LS by a small amount of ${ }^{252} \mathrm{Cf}$ that was accidentally introduced into both detectors during detector calibrations in October 2012. It is found that the source container did not have a tight seal due to a loose $\mathrm{O}$ ring. When the source was submerged in Gd-LS during source calibrations, Gd-LS seeped into the source container and a small amount of dissolved ${ }^{252} \mathrm{Cf}$ leaked into Gd-LS. Among the $~ 500$ day data sample the last 105 (79) days of data in the far (near) detector are contaminated by ${ }^{252} \mathrm{Cf}$. Thus the ${ }^{252} \mathrm{Cf}$ background removal criteria to be described later are applied to data taken during these periods. It is known that a ${ }^{252} \mathrm{Cf}$ decay emits 3.7 neutrons per fission on average with a mean energy of $2.1 \mathrm{MeV}$ per neutron, via $\alpha$ emission (96.9\%) and spontaneous fission (3.1\%).

\section{EVENT SELECTIONS}

Event selection criteria are applied to obtain IBD candidate events without distorting the spectral shape of IBD signal events. Because an IBD candidate requires a delayed signal from a neutron capture by Gd in Gd-LS, a fiducial volume naturally becomes the entire target region

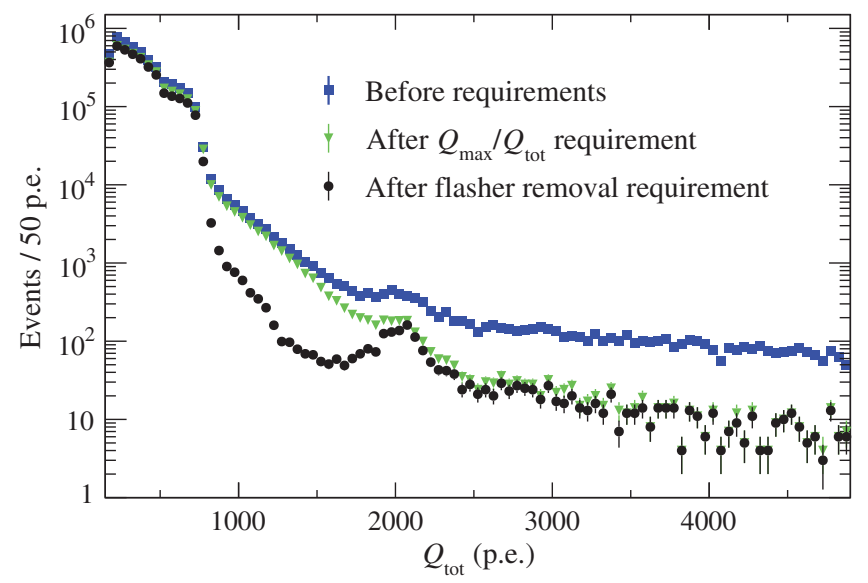

FIG. 12. $Q_{\text {tot }}$ distributions of events before applying any selection criterion (blue), applying $Q_{\max } / Q_{\text {tot }}<0.07$ requirement (green), and flashing PMT removal condition (black).

without a vertex position requirement. As a result, the detection efficiency is enhanced by some spill-in of IBD events.

Before applying prompt and delayed coincidence criteria, the following three preselection criteria are applied to all buffer-only triggered events: (i) $Q_{\max } / Q_{\text {tot }}<0.07$ where $Q_{\max }$ is the maximum charge of any single ID PMT, to eliminate external $\gamma$-ray events and flashing PMT events; (ii) an additional PMT hit timing and charge requirement of $Q_{\max } / Q_{\mathrm{tot}}<0.07$ where an extended timing window of -400 to $+800 \mathrm{~ns}$ is imposed to calculate $Q_{\text {tot }}$ and $Q_{\max }$ for this criterion, to eliminate events coming from remaining flashing PMTs effectively; (iii) timing veto criteria to reject events associated with the cosmic muons (a) if they are within a $1 \mathrm{~ms}$ window following a cosmic muon of $E_{\mu}>70 \mathrm{MeV}$, or of $20<E_{\mu}<70 \mathrm{MeV}$ for OD $N_{\text {hit }}>50$, or (b) if they are within a $700 \mathrm{~ms}(400 \mathrm{~ms}$, $200 \mathrm{~ms}$ ) window following a cosmic muon of $E_{\mu}>$ $1.6 \mathrm{GeV}(1.5-1.6 \mathrm{GeV}, 1.4-1.5 \mathrm{GeV})$ for the near detector, or within a $700 \mathrm{~ms}(500 \mathrm{~ms}, 200 \mathrm{~ms})$ window following a cosmic muon of $E_{\mu}>1.5 \mathrm{GeV}(1.2-1.5 \mathrm{GeV}, 1.0-1.2 \mathrm{GeV})$ for the far detector. As shown in Fig. 12, the selection criteria based on $Q_{\max } / Q_{\text {tot }}$ are efficient to eliminate external $\gamma$-ray events and flashing PMT events. Figure 13 shows a clean delayed signal of $\sim 8 \mathrm{MeV} \gamma$ rays from neutron captures on $\mathrm{Gd}$ after the preselection criteria and a large radioactive background against $2.2 \mathrm{MeV} \gamma$ rays from neutron captures on hydrogen.

The following criteria are applied to select IBD candidates: (iv) a prompt energy requirement of $0.7<E_{\mathrm{p}}<12 \mathrm{MeV}$; (v) a delayed energy requirement of $6<E_{\mathrm{d}}<12 \mathrm{MeV}$ where $E_{\mathrm{d}}$ is the energy of a delayed-like event; (vi) a time coincidence requirement of $2<\Delta t_{e^{+} n}<100 \mu \mathrm{s}$ where $\Delta t_{e^{+} n}$ is the time difference between the prompt-like and delayed-like events; (vii) a spatial coincidence requirement of $\Delta R<2.5 \mathrm{~m}$ where $\Delta R$ is the distance between vertices of the prompt-like and delayed-like events, to eliminate 


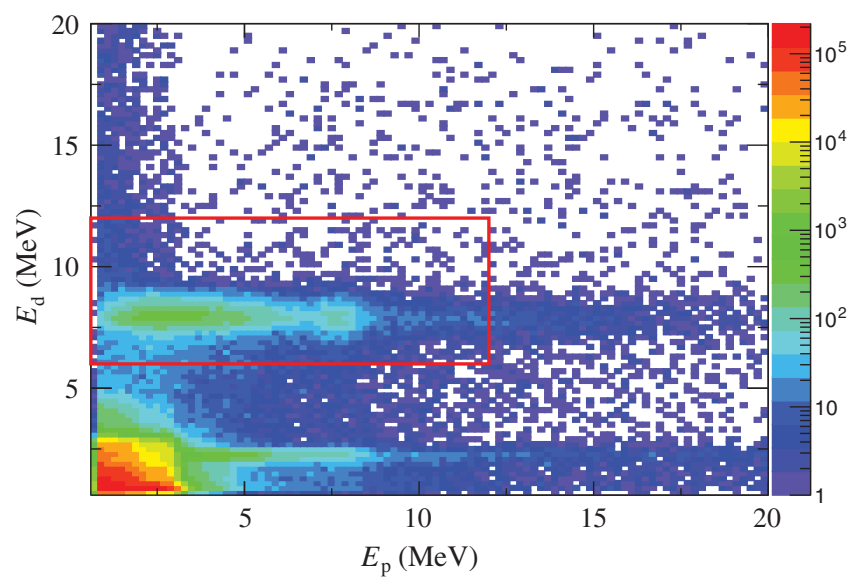

FIG. 13. Prompt versus delayed energies of IBD candidates after the preselection criteria using the 400 day data prior to the ${ }^{252} \mathrm{Cf}$ contamination. Neutron captures on $\mathrm{Gd}$ (red box) and $\mathrm{H}$ are clearly seen in the delayed energy distribution before full selection criteria. Accidental background events highly populate the region below prompt energy of $3 \mathrm{MeV}$.

remaining accidental backgrounds. The coincidence requirements of a delayed candidate are quite efficient for removing accidental backgrounds mostly in the low energy region of $E_{\mathrm{p}}<3 \mathrm{MeV}$.

The following multiplicity requirements are applied to remove events of a fast neutron, multiple neutrons, and the ${ }^{252} \mathrm{Cf}$ contamination background: (viii) a timing veto requirement for rejecting coincidence pairs (a) if they are accompanied by any preceding ID or OD trigger within a $100 \mu$ s window before their prompt candidate, (b) if they are followed by any subsequent ID-only trigger other than those associated with the delayed candidate within a $200 \mu \mathrm{s}$ window from their prompt candidates, (c) if they are followed by any subsequent ID and OD trigger within a $200 \mu$ s window from their prompt candidates, (d) if there are other subsequent pairs within the $500 \mu$ s interval, (e) if they are accompanied by any prompt candidate of $E_{\mathrm{p}}>$ $0.7 \mathrm{MeV}$ within a $300 \mu$ s preceding window or a $1 \mathrm{~ms}$ subsequent window, or (f) if they are accompanied by a prompt candidate of $E_{\mathrm{p}}>3 \mathrm{MeV}$ within a $10 \mathrm{~s}$ window and a distance of $40 \mathrm{~cm}$; (ix) a spatial veto requirement for rejecting coincidence pairs in the far detector only if the vertices of their prompt candidates are located in a cylindrical volume of $30 \mathrm{~cm}$ in radius, centered at $x=$ $+12.5 \mathrm{~cm}$ and $y=+12.5 \mathrm{~cm}$, and $-170<z<-120 \mathrm{~cm}$.

The criteria of (viii) (a), (b), (c), (d), and (e) eliminate events due to multiple neutrons or multiple interactions of a neutron with protons in the ID. They also eliminate the ${ }^{252} \mathrm{Cf}$ contamination background. The criteria (viii) (f) and (ix) are applied to eliminate the ${ }^{252} \mathrm{Cf}$ contamination background. The criterion (viii) (f) is useful for removing multiple neutron events from the ${ }^{252} \mathrm{Cf}$ decays. The criterion (ix) removes events from a region highly populated by events from decays of ${ }^{252} \mathrm{Cf}$ that is thought to have settled down at the bottom of the target of the far detector.

Applying the IBD selection criteria yields 31541 (290775) candidate events with $E_{\mathrm{p}}$ between 1.2 and $8.0 \mathrm{MeV}$ for a live time of 489.93 (458.49) days in the far (near) detector, in the period between August 2011 and January 2013. IBD events with $E_{\mathrm{p}}<1.2 \mathrm{MeV}$ include IBD events occurring in or near the target vessel wall that deposit positron kinetic energy in the wall without producing scintillation lights. These events are reconstructed to have visible energy near the positron annihilation energy of $1.02 \mathrm{MeV}$ and are not well reproduced by the MC prediction. The IBD signal loss by the $E_{\mathrm{p}}>1.2 \mathrm{MeV}$ requirement is roughly $2 \%$ in both detectors. The prompt events occurring near the target vessel wall could lose some of their energy to the nonscintillating target wall and lead to slight modifications of their prompt energies. However, the energy mismeasurement affects both the near and far detectors in an identical way and thus has a negligible effect on the results.

The magnitudes and spectral shapes of the remaining backgrounds are estimated using background enriched samples and are subtracted from the final IBD candidate samples.

\section{DETECTION EFFICIENCY}

The detection efficiency uncertainties are categorized into correlated and uncorrelated uncertainties between the near and far detectors. The correlated uncertainty is common to both near and far detectors and thus canceled out for the far-to-near relative measurement while the uncorrelated uncertainty remains with no cancellation. $\mathrm{An}$ individual detector efficiency is measured from an IBD signal enriched sample, and its uncertainty is given by a statistical uncertainty and uncorrelated and correlated systematic uncertainties. The detection efficiencies for common event selection criteria (i) to (viii) for both near and far detectors are assumed to be the same since both detectors are believed to have identical performances. Therefore, the weighted mean of near and far efficiencies for each selection criterion is taken to be the efficiency. The systematic error of the average efficiency is estimated from data and MC simulation. The IBD signal enriched samples are not large enough to find all of the uncorrelated systematic uncertainties by the difference of the measured detection efficiencies. Some of the uncorrelated systematic uncertainties are estimated from the possible difference in properties and performances between the two detectors if the IBD signal enriched sample is small. In this section, we present detection efficiencies and their uncertainties for the IBD signal events at $1.2<E_{\mathrm{p}}<8.0 \mathrm{MeV}$.

An expected number of IBD interactions is determined by reactor fluxes, an IBD cross section, and a total number of free protons in the target. The uncertainty of the IBD cross section from a theoretical calculation [21] is $0.13 \%$ and can be ignored by the relative measurement. 
The number of free protons in the target is estimated as $(1.189 \pm 0.003) \times 10^{30}$, based on the measurements of LAB density $\left(0.856 \pm 0.001 \mathrm{~g} / \mathrm{cm}^{3}\right)$ and target volume [14]. The uncorrelated systematic uncertainty of the number of free protons is $0.03 \%$, estimated from the measured volume difference of 41 between the near and far target vessels [12]. The correlated uncertainty is $0.5 \%$, estimated from the resolution of a densitometer.

The trigger efficiency is determined by the IBD signal loss due to the requirement of ID $N_{\text {hit }}>90$. The RENO Monte Carlo simulation, which is described later, does not reproduce the data $N_{\text {hit }}$ well due to the lack of realistic individual-channel simulation for the p.e. threshold and dark or noise hits. According to a comparison of $N_{\text {hit }}$ distribution between data and $\mathrm{MC}$ simulation, a $\mathrm{MC}$ equivalent requirement of $N_{\text {hit }}>84$ is found to accept a buffer-only trigger. Using the $\mathrm{MC}$ equivalent hit requirement, the trigger efficiency for the IBD signal excluding spill-in events in the near (far) detector is estimated as $99.77 \pm 0.05 \%$ $(99.78 \pm 0.13 \%)$ where spill-in events are events that occur outside the target and produce a neutron capture on Gd in the target. The trigger efficiency is also measured for the events at the detector center using radioactive sources and is consistent with the MC simulation result within the uncertainty. The position dependent DAQ inefficiency contributes to the inefficiency near the trigger threshold below $\sim 0.8 \mathrm{MeV}$. Our measured trigger efficiency using a ${ }^{137} \mathrm{Cs}$ source $(E=0.63 \mathrm{MeV})$ is roughly $50 \%$ at the threshold energy of $0.5-0.6 \mathrm{MeV}$ and almost $100 \%$ at $0.8 \mathrm{MeV}$. The uncorrelated systematic uncertainty of the trigger efficiency is estimated as $0.01 \%$ from the difference between near and far efficiencies. The correlated uncertainty of the trigger efficiency is estimated as $0.01 \%$ from the ambiguity in finding a MC equivalent $N_{\text {hit }}$ threshold.

The efficiency of the $Q_{\max } / Q_{\mathrm{tot}}<0.07$ criterion is obtained using an IBD candidate sample of almost no accidental background events that are selected by a stringent spatial-correlation requirement of $\Delta R<0.3 \mathrm{~m}$. The $Q_{\max } / Q_{\text {tot }}$ distribution of this sample predicts an expected IBD signal loss in the region of $Q_{\max } / Q_{\text {tot }}>0.07$, by extrapolating from the region of $Q_{\max } / Q_{\text {tot }}<0.07$ using an expected shape of MC simulation. The efficiency is estimated as $99.99 \%$ using the measured values of $99.996 \pm$ 0.003 (stat.) \% and $99.98 \pm 0.01$ (stat.) $\%$ for the near and far detectors, respectively. The correlated uncertainty is estimated from the ambiguity of the extrapolation and found to be $0.01 \%$. The uncorrelated systematic uncertainty is estimated from the obtained efficiency difference between the near and far detectors and found to be $0.02 \%$.

The efficiency of the prompt energy requirement is obtained from the fraction of events in the region of $1.2<$ $E_{\mathrm{p}}<8.0 \mathrm{MeV}$ relative to total IBD events and estimated as $98.77 \%$ using the measured values of $98.78 \pm 0.03$ (stat.) $\%$ and $98.66 \pm 0.09$ (stat.) $\%$ for the near and far detectors, respectively. The uncorrelated systematic uncertainty is estimated to be $0.01 \%$ by varying the energy threshold according to the energy-scale difference of $0.15 \%$ between the near and far detectors. The correlated uncertainty is estimated to be $0.09 \%$ by varying the energy threshold according to the energy-scale uncertainty of $1.0 \%$.

The efficiency of the delayed energy requirement is determined by the fraction of delayed events in the region of $6<E_{\mathrm{d}}<12 \mathrm{MeV}$ out of total delayed events of neutron capture on Gd. An IBD event enriched sample is used for the efficiency estimation and obtained by requiring IBD candidates to have $4<E_{\mathrm{p}}<8 \mathrm{MeV}$ to eliminate accidental and fast neutron backgrounds and $3.5<E_{\mathrm{d}}<12 \mathrm{MeV}$ to accept lower energy delayed events. According to a MC simulation, $1.16 \%$ of the total delayed events are found at $E_{\mathrm{d}}<3.5 \mathrm{MeV}$. With this correction, the efficiency is estimated as $92.14 \%$ using the measured values of $92.15 \pm$ 0.08 (stat.) $\%$ and $92.05 \pm 0.26$ (stat.) $\%$ from the near and far IBD event enriched samples, respectively. The correlated uncertainty is estimated to be $0.50 \%$ by considering the $\mathrm{MC}$ simulation correction uncertainty below $3.5 \mathrm{MeV}$ and varying the energy scale by its uncertainty of $1.0 \%$. The uncorrelated systematic uncertainty is estimated to be $0.05 \%$ by changing the delayed energy requirement by $\pm 0.15 \%$, the energy scale difference between the near and far detectors.

The Gd capture fraction is measured by the ratio of neutron captures on $\mathrm{Gd}$ to total neutron captures on $\mathrm{Gd}$ or $\mathrm{H}$ using ${ }^{252} \mathrm{Cf}$ source data that are taken at the detector center. The effects of spill-in/out events at the target boundary are treated separately and described later. $\mathrm{A}^{252} \mathrm{Cf}$ source sample including $\mathrm{H}$ capture delayed events is obtained by requiring prompt and delayed event pairs satisfying $4<E_{\mathrm{p}}<12 \mathrm{MeV}$ and $1.5<E_{\mathrm{d}}<12 \mathrm{MeV}$, respectively. An additional neutron candidate of $1.5<E_{\mathrm{d}}<3 \mathrm{MeV}$ or $6<E_{\mathrm{d}}<10 \mathrm{MeV}$ within $200 \mu$ s from the prompt event of a coincidence pair is required to ensure the delayed events are neutron capture events originating from ${ }^{252} \mathrm{Cf}$ decay. The obtained delayedenergy distributions show a good agreement between near and far detectors as shown in Fig. 14. We obtain the Gd capture fraction by the ratio of the $n$-Gd events with $E_{\mathrm{d}}>$ $3.5 \mathrm{MeV}$ to the total neutron capture events with $E_{\mathrm{d}}>1.5 \mathrm{MeV}$. A MC simulation finds contributions of neutron captures on $\mathrm{Gd}$ below $3.5 \mathrm{MeV}$ and of neutron captures on $\mathrm{H}$ below $1.5 \mathrm{MeV}$. With these contributions the Gd capture fraction is estimated as $85.45 \%$ using the measured values of $85.49 \pm 0.03$ (stat.) $\%$ and $85.40 \pm$ 0.07 (stat.)\% from the near and far data, respectively, while it is obtained as $88.41 \%$ from the MC simulation. The measured values of the Gd capture fractions are constant in time within their uncertainties. The correlated uncertainty is estimated as $0.47 \%$ mostly due to the uncertainty of the $n-\mathrm{Gd}$ capture cross section [22]. The uncorrelated systematic uncertainty is estimated as $0.1 \%$ due to the difference of $\mathrm{Gd}$ concentration between the near and far detectors. The difference is estimated to be less than $0.1 \%$ from the precision of dividing the Gd-LS equally for the two detectors. 


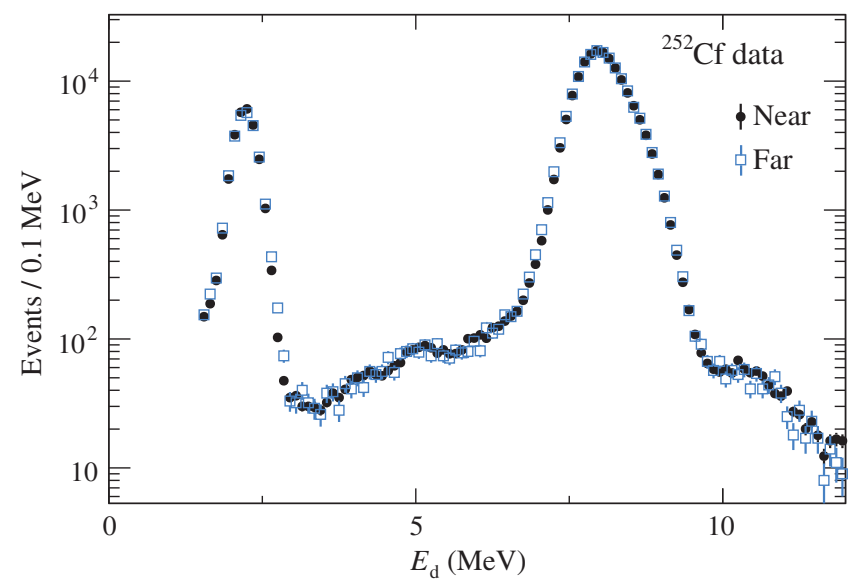

FIG. 14. Comparison of delayed energy distribution of neutron captures on $\mathrm{H}$ or $\mathrm{Gd}$ using ${ }^{252} \mathrm{Cf}$ source data. To obtain the accurate delayed energy spectra the ${ }^{252} \mathrm{Cf}$ source data were taken for 8 (2) h on April 2014 (January 2013) for the near (far) detector.

The efficiency of the time coincidence requirement is determined by the fraction of IBD events with $2<\Delta t_{e^{+}{ }_{n}}<$ $100 \mu$ s out of total IBD events. An IBD signal enriched sample is obtained by requiring IBD candidate events with $4<E_{\mathrm{p}}<8 \mathrm{MeV}$ in order to eliminate accidental backgrounds. Figure 15 shows $\Delta t_{e^{+} n}$ distributions of the neutron capture on Gd for the near and far IBD signal enriched samples. The fits to data are made by two exponential functions plus a constant that are multiplied by one minus an exponential function. The distributions are well described by

$$
\begin{aligned}
N(t)= & {\left[p_{0} \exp \left(-t / p_{1}\right)+p_{2} \exp \left(-t / p_{3}\right)+C\right] } \\
& \times\left[1-p_{4} \exp \left(-t / p_{5}\right)\right],
\end{aligned}
$$

where the parameters of $p_{0}, p_{1}, p_{4}, p_{5}$, and $C$ are determined by a fit to the data. Note that the parameters $p_{2}$ and $p_{3}$ of the second exponential function are estimated using the MC simulation. The first exponential function represents the capture time distribution of the IBD events without the spill-in events in the target region. The second exponential function is necessary to extract the contribution of the delayed events originating from the vicinity of the target vessel wall. The delayed signal of a spill-in event tends to have a longer capture time because of its drift from the $\gamma$ catcher to the target. The third exponential function describes the rising capture time behavior below $\sim 10 \mu \mathrm{s}$ where the IBD neutron is thermalized before capture. The efficiency in the central region is obtained by the fraction of IBD events with $2<\Delta t_{e^{+} n}<100 \mu \mathrm{s}$ out of the total IBD events that are estimated from the fitted mean value of capture time using the first exponential function. The measured capture time values for non-spill-in events are consistent between near and far detectors. To obtain the efficiency of non-spill-in events, a MC simulation is used to estimate the contribution of spill-in events inside the target. The efficiency is estimated as $96.59 \%$ using the measured values of $96.60 \pm 0.04$ (stat.)\% and

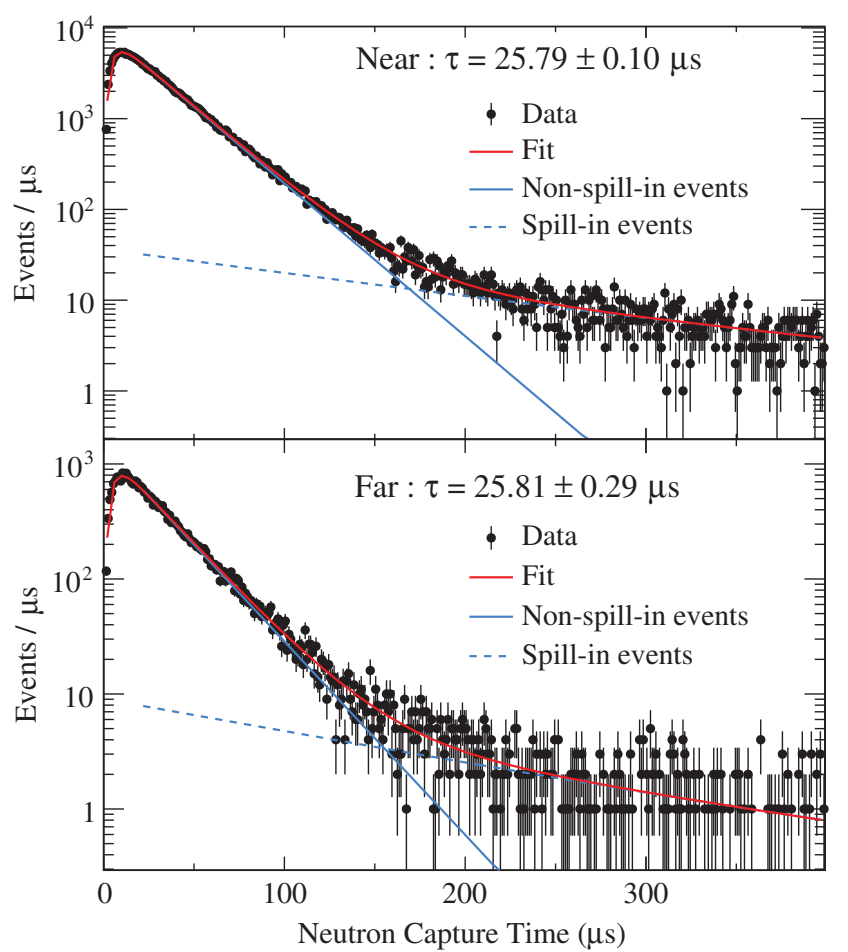

FIG. 15. Measured time distributions of neutron capture on Gd. The red solid curves are the fits to the data, and the solid (dotted) blue lines are the fitted capture time distributions of the IBD events in the central (outer) region. The mean capture times in the central region of the near and far detectors are found to be consistent with each other. The outer delayed events originating from the vicinity of the target vessel walls show longer capture time values. A total of 1400 live days of data is used in these plots for more precise measurements.

$96.57 \pm 0.10$ (stat.) $\%$ from the near and far data, respectively. The correlated uncertainty is estimated to be $0.26 \%$ from the uncertainty associated with a rising capture time of a delayed signal. The uncorrelated systematic uncertainty is estimated as $0.01 \%$ from the uncertainty of $\mathrm{Gd}$ concentration difference, $\sim 0.1 \%$, between the near and far detectors.

The efficiency of the spatial coincidence requirement, $\Delta R<2.5 \mathrm{~m}$, is obtained from IBD candidates with $Q_{\max } / Q_{\text {tot }}<0.02$. The efficiency is estimated as $100.00 \%$ using the measured values of $99.99 \pm 0.01$ (stat.) $\%$ and $100.00 \pm 0.01$ (stat.)\% from the near and far data, respectively, assuming $100 \%$ at $\Delta R<5 \mathrm{~m}$. The correlated uncertainty is estimated as $0.02 \%$ based on changing the $\Delta R$ requirement by the resolution of reconstructed vertex, $0.3 \mathrm{~m}$. The uncorrelated systematic uncertainty is estimated as $0.02 \%$ from the efficiency difference between the near and far detectors.

The spill-in events enhance the detection efficiency of IBD signals in the target because of additional IBD signals occurring outside the target but with its neutron capture by $\mathrm{Gd}$ in the target. On the other hand, the reactor $\bar{\nu}_{e}$ interaction occurring in the target edge may be lost because of a neutron capture in the $\gamma$-catcher region by $\mathrm{H}$. Such an event 
TABLE IV. Average detection efficiencies and their uncertainties of common selection criteria that are applied to both near and far detectors for the IBD candidates. The total detection efficiency is the statistical error weighted mean of the total detection efficiencies of the near and far detectors.

\begin{tabular}{lccc}
\hline \hline & $\begin{array}{c}\text { Efficiency } \\
{[\%]}\end{array}$ & $\begin{array}{c}\text { Uncorrelated } \\
{[\%]}\end{array}$ & $\begin{array}{c}\text { Correlated } \\
{[\%]}\end{array}$ \\
\hline IBD cross section & $\ldots$ & $\ldots$ & 0.13 \\
Target protons & $\ldots$ & 0.03 & 0.5 \\
Trigger efficiency & 99.77 & 0.01 & 0.01 \\
$Q_{\text {max }} / Q_{\text {tot }}$, & 100.00 & 0.02 & 0.01 \\
$\quad$ antiflasher & & & \\
Prompt energy & 98.77 & 0.01 & 0.09 \\
Delayed energy & 92.14 & 0.05 & 0.50 \\
Gd capture fraction & 85.48 & 0.10 & 0.47 \\
Time coincidence & 96.59 & 0.01 & 0.26 \\
Spatial correlation & 100.00 & 0.02 & 0.02 \\
Spill-in & 102.00 & 0.04 & 0.61 \\
Total detection & 76.47 & 0.13 & 1.09 \\
$\quad$ efficiency & & & \\
\hline \hline
\end{tabular}

loss is accounted for in the delayed energy requirement efficiency. The enhanced detection efficiency due to the spillin events is estimated as $102.00 \%$ using the measured values of $102.02 \%$ and $101.98 \%$ using near and far MC simulation, respectively. The uncorrelated systematic uncertainty is estimated as $0.04 \%$ due to differences of the Gd concentration and the acrylic wall thickness of the target vessel between the near and far detectors. The correlated uncertainty is estimated as $0.61 \%$ based on the delayed time distribution of spill-in events at $\Delta t_{e^{+} n}>200 \mu$ s deviating from that of IBD events in the target.

The detection efficiencies of selection criteria that are applied to both near and far detectors are summarized in
Table IV. Their identical performances minimize the uncorrelated systematic uncertainties and allow cancellation of the correlated systematic uncertainties for the ratio measurement. The measured efficiencies in total are $76.51 \pm$ 0.10 (stat.) $\%$ and $76.20 \pm 0.30$ (stat.) $\%$ for the near and far detectors, respectively, with common uncorrelated $(0.13 \%)$ and correlated $(1.09 \%)$ uncertainties. The average efficiency for each selection criterion is calculated as an error weighted mean of the near and far measured values. The error weighting is done using a statistical error. The average efficiency in total is obtained as $76.47 \pm 0.16 \%$ where the error is calculated by adding all the selection-efficiency statistical and uncorrelated systematic errors in quadrature. The near and far detection efficiencies differ from the total average efficiency by $0.04 \%$ (near) and $0.27 \%$ (far), respectively, and the differences are reasonably within the statistical errors. IBD signal enriched samples for some selection criteria, due to their small sizes, do not allow direct checks of the estimated uncorrelated uncertainties by the difference of the measured near and far efficiencies. With larger IBD signal enriched samples, especially in the far detector, the uncorrelated systematic uncertainties are expected to be improved in the future. In the rate and spectral fit the uncertainty of the far-to-near detection efficiency ratio is taken into account for one of the pull parameter uncertainties. We obtain the uncorrelated uncertainty of the efficiency ratio as $0.21 \%$ from combining the uncorrelated uncertainty and the weighted statistical errors of the measured values. We take $0.20 \%$ as the value of the efficiency ratio uncertainty, the same as our published result [11] because both values give essentially identical systematic errors.

Among the IBD selection criteria, the muon and multiplicity timing veto requirements are applied differently to the near and far detectors, and thus introduce no correlation at all between the detectors. The IBD signal losses due to the muon

TABLE V. Summary of the IBD signal loss due to timing veto criteria. The criterion with (*) is applied only to the ${ }^{252} \mathrm{Cf}$ contaminated data of $\sim 100$ days. Note that the uncertainties are treated as being fully uncorrelated between the near and far detectors.

\begin{tabular}{|c|c|c|}
\hline & Signal loss [\%] & Signal loss $[\%]$ \\
\hline Timing veto criteria & Near & Far \\
\hline (i) Timing criteria associated with muon & $21.558 \pm 0.003$ & $11.133 \pm 0.003$ \\
\hline (ii) Adjacent IBD pair within $500 \mu \mathrm{s}$ & 0 & 0 \\
\hline $\begin{array}{l}\text { (iii) IBD candidate accompanied by any trigger within } \\
100 \mu \text { s preceding time window }\end{array}$ & $4.672 \pm 0.001$ & $1.309 \pm 0.001$ \\
\hline $\begin{array}{l}\text { (iv) IBD candidate accompanied by ID-only trigger within } \\
200 \mu \text { s subsequent time window }\end{array}$ & $1.134 \pm 0.001$ & $1.424 \pm 0.001$ \\
\hline $\begin{array}{l}\text { (v) IBD candidate accompanied by prompt candidate within } \\
300 \mu \text { s preceding time window }\end{array}$ & $0.605 \pm 0.001$ & $0.163 \pm 0.001$ \\
\hline $\begin{array}{l}\text { (vi) IBD candidate accompanied by prompt candidate within } \\
1 \mathrm{~ms} \text { subsequent time window }\end{array}$ & $0.258 \pm 0.001$ & $0.638 \pm 0.001$ \\
\hline $\begin{array}{l}\text { (vii) IBD candidate accompanied by ID and OD triggers } \\
\text { within } 200 \mu \text { s subsequent time window }\end{array}$ & $0.408 \pm 0.001$ & $0.069 \pm 0.001$ \\
\hline $\begin{array}{l}\text { (viii) IBD candidate accompanied by prompt candidate } \\
(>3 \mathrm{MeV}) \text { occurring within } 10 \mathrm{~s} \text { and } 40 \mathrm{~cm}(*)\end{array}$ & $0.491 \pm 0.006$ & $0.388 \pm 0.020$ \\
\hline Combined IBD signal loss & $27.364 \pm 0.007$ & $14.691 \pm 0.021$ \\
\hline
\end{tabular}


veto requirements are $21.558 \%$ and $11.133 \%$ for the near and far detectors, respectively, with both of their uncertainties less than $0.03 \%$. The total IBD signal loss due to the timing veto requirements is $27.364 \pm 0.007 \%(14.691 \pm 0.021 \%)$ for the near (far) detector as summarized in Table V.

\section{REMAINING BACKGROUND ESTIMATIONS AND THEIR UNCERTAINTIES}

The remaining backgrounds after event selection requirements are subtracted from the final IBD candidate sample. The following subsections describe how to obtain the spectral shapes and rates of the remaining backgrounds. Since the rates and shapes of all the remaining backgrounds are measured from background enriched samples, their uncertainties are expected to be further reduced with more data.

\section{A. Accidental background}

Most of the accidental background events are eliminated by requiring timing and spatial coincidence between the prompt-like and delayed-like events. An accidental background sample is obtained by requiring temporal dissociation between prompt- and delayed-like events, i.e., $\Delta t_{e^{+} n}>1 \mathrm{~ms}$ for the IBD sample with no $\Delta R$ requirement. The prompt energy spectra of the accidental backgrounds of the near and far detectors are shown in Figs. 16(a) and 16(b). The energy-bin-uncorrelated uncertainty in the accidental background spectrum is obtained from the statistical error of the background enriched sample and estimated as 0.02 (0.01) events per day for the near (far) detector.

The remaining rate in the final sample is estimated by measuring the rate of random spatial associations in the IBD signal region of $\Delta R<2.5 \mathrm{~m}$, extrapolated from the background dominant region of $\Delta R>1.75 \mathrm{~m}$ using the $\Delta R$ distribution of the accidental background spectrum as shown in Fig. 16(c). The energy-bin-correlated uncertainty is obtained from the fitting error and estimated as 0.08 (0.03) events per day for the near (far) detector. The obtained accidental-background rates are $6.89 \pm 0.09$ (near) and $0.97 \pm 0.03$ (far) events per day.

\section{B. Fast neutron background}

The fast neutron background rate in the final IBD candidate sample is estimated by being extrapolated from the background dominant energy region of $12<E_{\mathrm{p}}<$ $40 \mathrm{MeV}$ to the IBD signal region of $1.2<E_{\mathrm{p}}<8.0 \mathrm{MeV}$, assuming a flat spectrum of the background as shown in Fig. 17. A fast neutron enriched sample is obtained by selecting IBD candidates that are accompanied by any prompt candidates of $E_{\mathrm{p}}>0.7 \mathrm{MeV}$ within a $1 \mathrm{~ms}$ subsequent window. The prompt events of this sample show a distribution consistent with a flat spectrum in the IBD signal region as shown in Fig. 18. The background rate uncertainty is obtained from the fitting error of the flat spectrum and estimated as $0.03(0.02)$ events per day for the near (far)
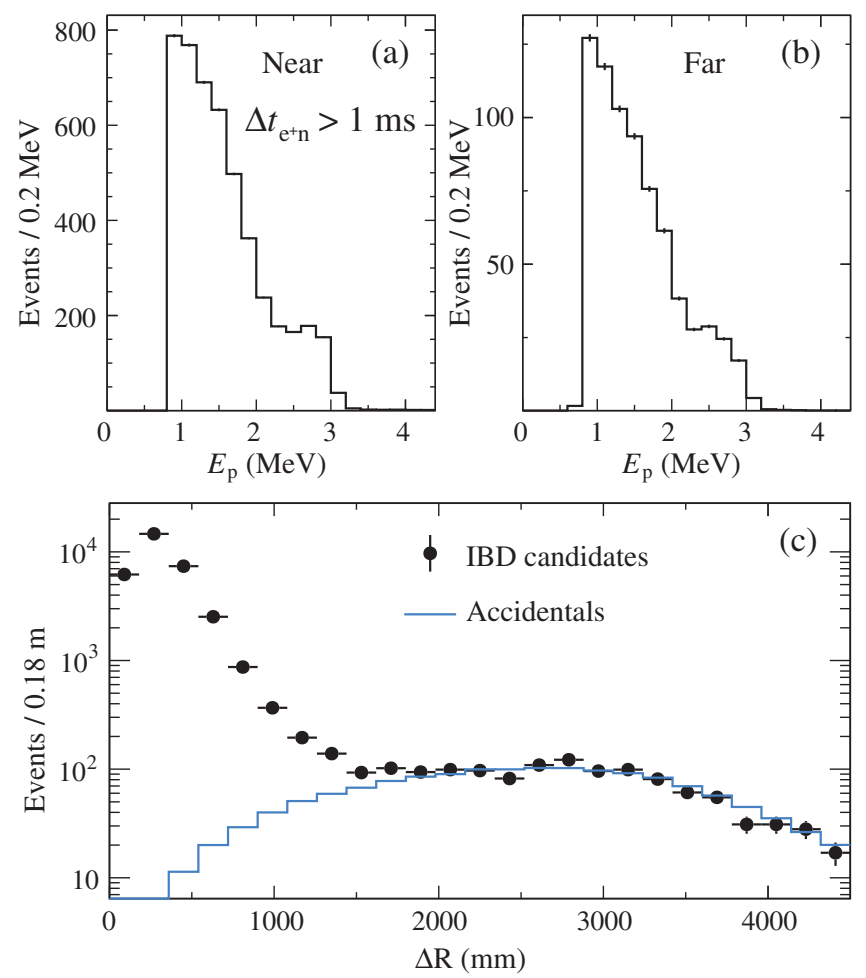

FIG. 16. (a) and (b) Prompt energy spectra of accidental backgrounds obtained from accidental background enriched samples that are selected by temporal association larger than $1 \mathrm{~ms}$. They are normalized to the remaining background. The error bars represent statistical and spectral shape uncertainties. (c) Spatial correlation $(\Delta R)$ distribution of IBD candidates with no $\Delta R$ requirement. The amount of accidental background is obtained by a fit to data using the $\Delta R$ distribution from the accidental background enriched sample.

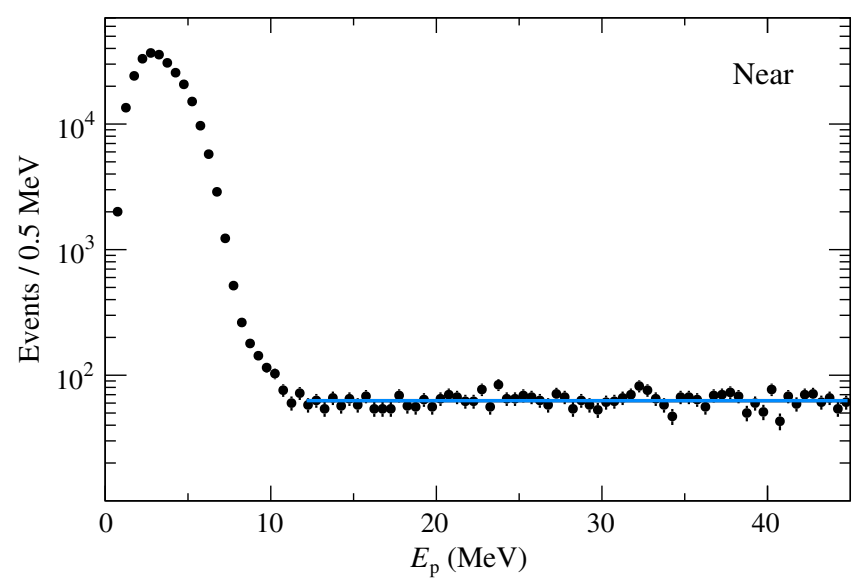

FIG. 17. Prompt energy spectrum of IBD candidates including a flat fast neutron spectrum at $E_{\mathrm{p}}>12 \mathrm{MeV}$. The fast neutron background rate in the IBD candidates is estimated by extrapolating from the background dominant region assuming a flat spectrum of the background. 


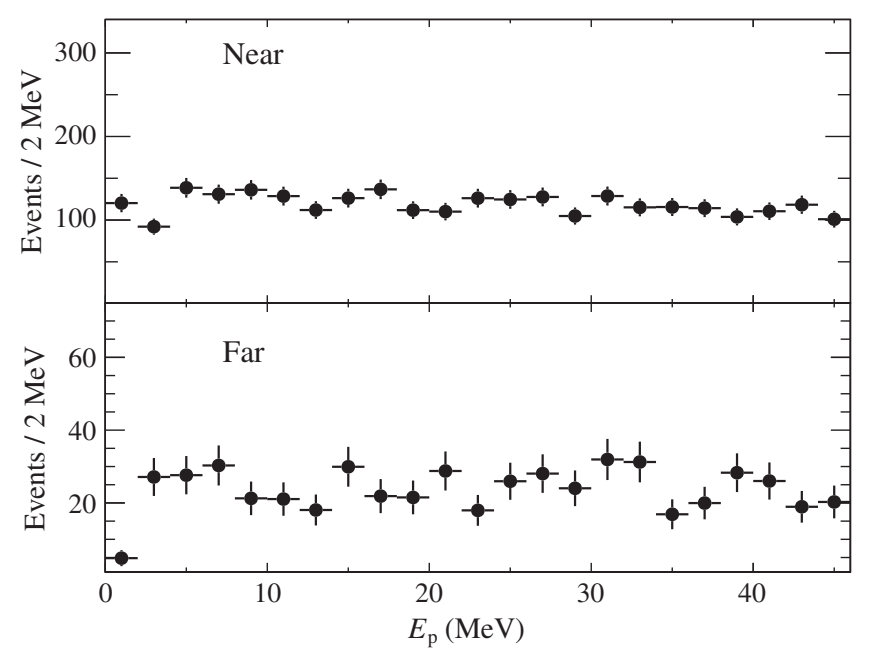

FIG. 18. Prompt energy spectra of fast neutron enriched samples in the near and far detectors.

detector. The assumption of the flat background spectrum in the signal region is checked and validated by a fast neutron background enriched sample. The spectral shape uncertainty of the fast neutron background includes a possible deviation from the flat spectrum and is estimated as $0.02(0.01)$ events per day for the near (far) detector. In order to estimate the deviation, the background dominant region in Fig. 18 is fitted with a first order polynomial as an alternative model. The remaining fast neutron background rates are $2.28 \pm 0.04$ (near) and $0.48 \pm 0.02$ (far) events per day.

Some of the fast neutrons lose most of their kinetic energy before reaching the target or $\gamma$-catcher regions and produce neutron capture events. These neutron capture events are easily paired with a prompt-like event to contribute to accidental backgrounds. Those backgrounds are eliminated if any buffer and veto trigger occurs in a $100 \mu$ s window following a prompt candidate.

\section{Cosmogenic ${ }^{9} \mathrm{Li} /{ }^{8} \mathrm{He}$ background}

The spectral shape of the ${ }^{9} \mathrm{Li} /{ }^{8} \mathrm{He}$ background is measured using a sample of IBD-like pairs that are produced within $500 \mathrm{~ms}(400 \mathrm{~ms})$ by energetic muons of $E_{\mu}>$ $1.6 \mathrm{GeV}(>1.5 \mathrm{GeV})$ for the near (far) detector. The distribution of time difference between an energetic muon and a subsequent IBD candidate is shown in Fig. 19. Based on their observed spectra, the shortest decay time component is found to be the muon-induced accidental background, and the ${ }^{9} \mathrm{Li} /{ }^{8} \mathrm{He}$ background follows after it. The IBD signals are temporally uncorrelated with muon events, and their time differences are distributed according to the IBD rate. The measured mean decay time of $\sim 250 \mathrm{~ms}$ indicates the predominant production of ${ }^{9} \mathrm{Li}$ over ${ }^{8} \mathrm{He}$.

The measured ${ }^{9} \mathrm{Li} /{ }^{8} \mathrm{He}$ background shapes as shown in Fig. 20 are obtained by subtracting the energy spectra of the IBD signal and the muon-induced accidental background from those of the ${ }^{9} \mathrm{Li} /{ }^{8} \mathrm{He}$ background enriched samples.

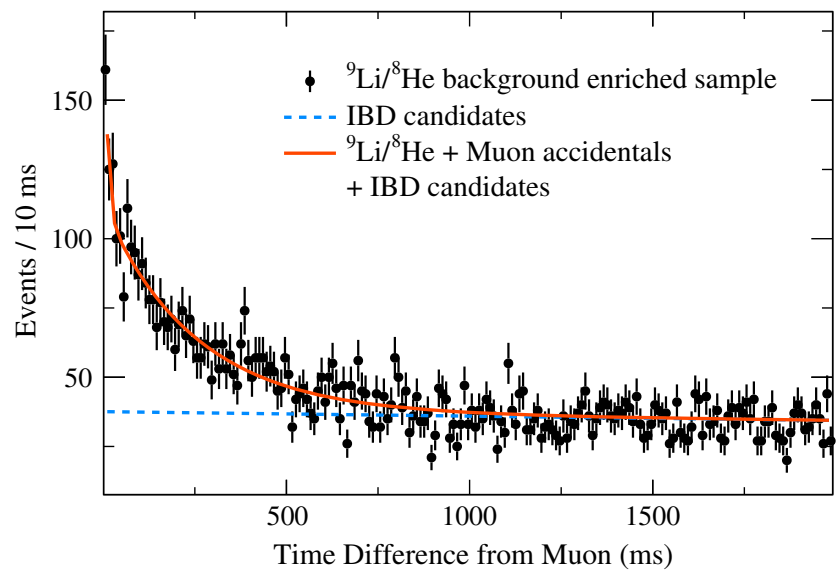

FIG. 19. Decay time distribution of the IBD-like pairs from their preceding energetic muons from a total of 1100 live days of the ${ }^{9} \mathrm{Li} /{ }^{8} \mathrm{He}$ background enriched sample in the far detector. The ${ }^{9} \mathrm{Li} /{ }^{8} \mathrm{He}$ background is clearly seen with a measured mean decay time of $\sim 250 \mathrm{~ms}$ while muon-induced accidental background events are observed right after their preceding muons.

The size of the IBD signal and the muon induced accidental background are determined by a fit to the decay time distribution using three exponential functions. The spectral

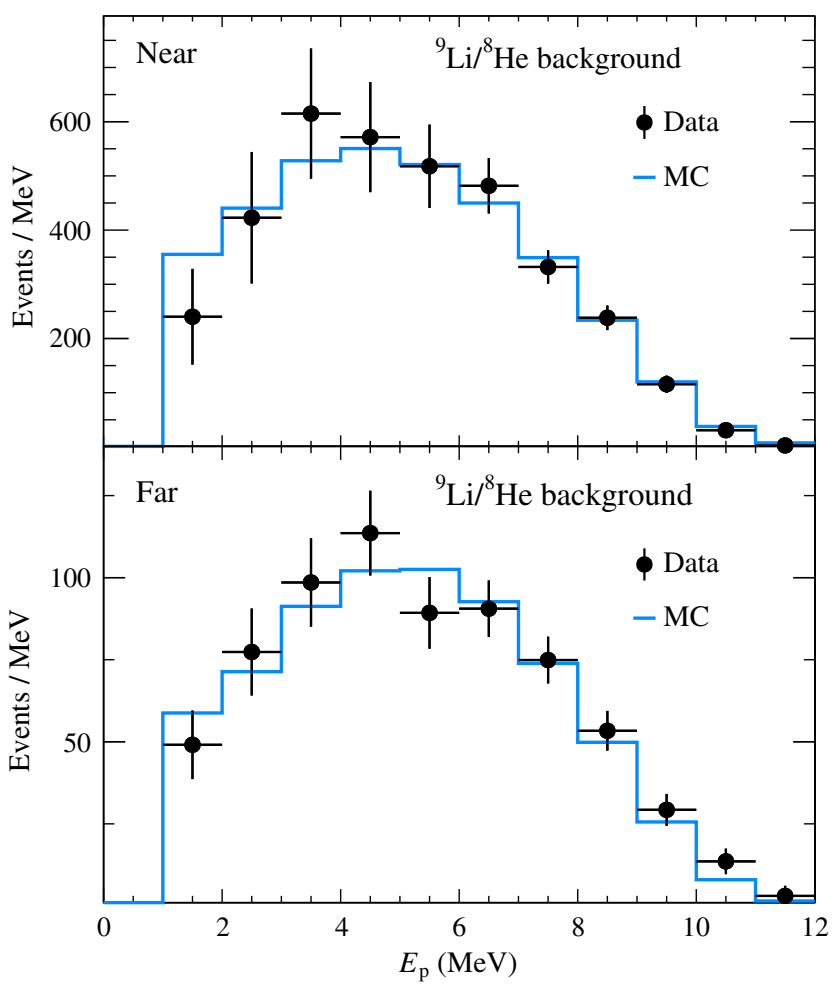

FIG. 20. Measured ${ }^{9} \mathrm{Li} /{ }^{8} \mathrm{He}$ background spectra that are obtained from the enriched samples of 1100 live days of data after subtracting the IBD signal and the muon induced accidental background from those of the enriched sample. The MC simulation ${ }^{9} \mathrm{Li} /{ }^{8} \mathrm{He}$ background spectra (blue histograms) are overlaid with data where the relative fractions between ${ }^{9} \mathrm{Li}$ and ${ }^{8} \mathrm{He}$ are obtained from the fits to the data. 


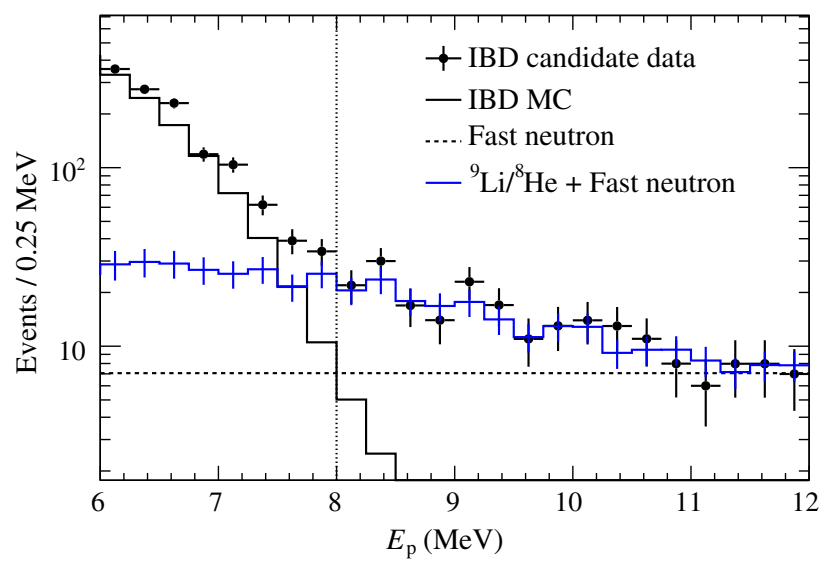

FIG. 21. Estimation of the remaining ${ }^{9} \mathrm{Li} /{ }^{8} \mathrm{He}$ background rate in the signal region using the measured rate in the background dominant region of $E_{\mathrm{p}}>8 \mathrm{MeV}$ in the far detector. The background rate in the signal region of $E_{\mathrm{p}}<8 \mathrm{MeV}$ is estimated by extrapolating from the background dominant region using the measured background spectrum.

shape uncertainty comes from statistical uncertainty of the ${ }^{9} \mathrm{Li} /{ }^{8} \mathrm{He}$ background enriched sample because of the subtraction and, therefore, is expected to be reduced by more data. The ${ }^{9} \mathrm{Li}$ and ${ }^{8} \mathrm{He}$ background shapes are also obtained from MC simulation for comparison. The relative fraction between ${ }^{9} \mathrm{Li}$ and ${ }^{8} \mathrm{He}$ is determined by a fit to the measured ${ }^{9} \mathrm{Li} /{ }^{8} \mathrm{He}$ spectrum. The estimated ${ }^{8} \mathrm{He}$ component is $13.6 \pm$ $3.9 \%(1.1 \pm 1.6 \%)$ for the near (far) detector. The difference of the ${ }^{8} \mathrm{He}$ components between the two detectors might be related to their different overburdens.

The background rate in the IBD signal region of $E_{\mathrm{p}}<8 \mathrm{MeV}$ is estimated by extrapolating from the background dominant region of $E_{\mathrm{p}}>8 \mathrm{MeV}$ using the measured background spectrum as shown in Fig. 21. The background rate in the region of $E_{\mathrm{p}}>8 \mathrm{MeV}$ is estimated by a fit to the IBD candidate data using the measured ${ }^{9} \mathrm{Li} /{ }^{8} \mathrm{He}$ background spectrum, the measured fast neutron background, and the MC IBD expectation. The energy-binuncorrelated spectral uncertainty is obtained from the measured ${ }^{9} \mathrm{Li} /{ }^{8} \mathrm{He}$ spectral uncertainty and is estimated as $0.61(0.07)$ events per day for the near (far) detector. The energy-bin-correlated uncertainty is obtained from the fit error of the background rate in the region of $E_{\mathrm{p}}>8 \mathrm{MeV}$ and estimated as $0.55(0.22)$ events per day for the near (far) detector. The estimated ${ }^{9} \mathrm{Li} /{ }^{8} \mathrm{He}$ background rates are $8.36 \pm 0.82$ (near) and $1.54 \pm 0.23$ (far) events per day.

\section{D. ${ }^{252} \mathrm{Cf}$ contamination background}

The amount of the initial ${ }^{252} \mathrm{Cf}$ contamination is estimated as $0.49 \pm 0.14 \mathrm{mBq}(4.51 \pm 0.94 \mathrm{mBq})$ for the near (far) detector. The estimation is made based on the rejected and remaining samples after event selection requirements (viii) and (ix). This background has a half-life of 2.7 years.
Most multiple neutron events coming from the ${ }^{252} \mathrm{Cf}$ contamination are eliminated by the stringent multiplicity requirements of no trigger or no event near an IBD event. After applying the requirements, $99.9 \%$ of the background events in the far detector is eliminated with a signal loss of $8.0 \pm 0.2 \%$. No remaining ${ }^{252} \mathrm{Cf}$ contamination background events are observed in the near detector. The remaining background rate and shape are obtained from the ${ }^{252} \mathrm{Cf}$ contamination candidate events that are accompanied by an additional event within a $10 \mathrm{~s}$ window and a distance of $40 \mathrm{~cm}$ from an IBD prompt event. Three different shape components of the background spectrum are found in this sample. They are two Gaussian-like spectral shapes peaking at 2.2 and $11.0 \mathrm{MeV}$ and a spectral shape peaking at $1 \mathrm{MeV}$ and falling rapidly up to $4 \mathrm{MeV}$ as shown in Fig. 22. It is not understood why there are three spectral shapes with different time correlations between an IBD prompt event and an associated event. The shape of the $11 \mathrm{MeV}(1 \mathrm{MeV})$ peaked component is obtained from a sample that is selected by requiring a time and spatially correlated event of $E>3 \mathrm{MeV}$ after (before) an IBD event. The associated event is likely due to the multiple neutrons or the prompt fission gammas from a ${ }^{252} \mathrm{Cf}$ decay. The shape of the $2.2 \mathrm{MeV}$ peaked component is obtained from a sample that is selected by requiring a time and spatially correlated event of $E<3 \mathrm{MeV}$ before an IBD event. The remaining ${ }^{252} \mathrm{Cf}$ background spectrum in the far detector is shown in Fig. 22.

The rate of the $11 \mathrm{MeV}$ peaked component is estimated by fitting the $E_{\mathrm{p}}$ distribution of the prompt events with no $E_{\mathrm{p}}$ requirement of the IBD event candidates. The $E_{\mathrm{p}}$ distribution is fit with the $11 \mathrm{MeV}$ peaked component spectrum and a flat fast neutron spectrum above $12 \mathrm{MeV}$, where the ${ }^{252} \mathrm{Cf}$ background is dominant. The other two component rates are estimated from the ${ }^{252} \mathrm{Cf}$ background dominant samples that are used for obtaining their component shapes. The remaining ${ }^{252} \mathrm{Cf}$ contamination background rate is estimated as $0.14 \pm 0.03$ events per day

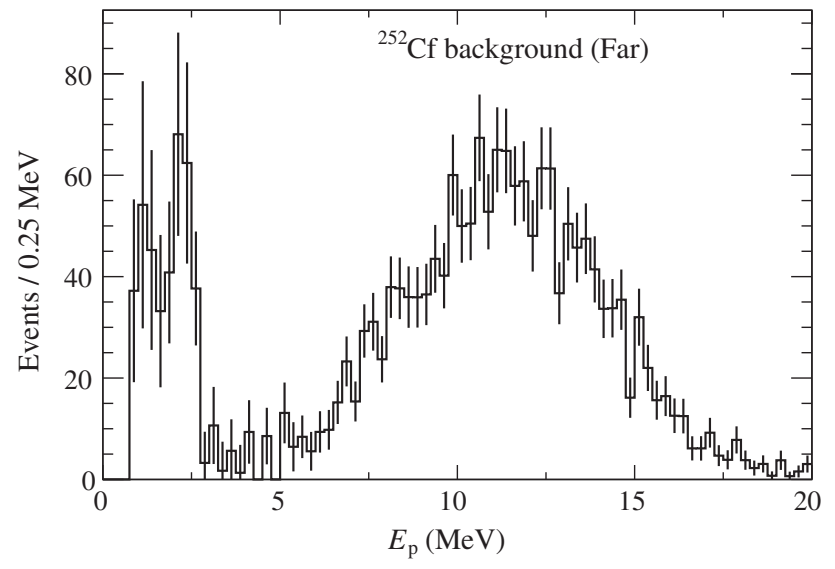

FIG. 22. ${ }^{252} \mathrm{Cf}$ background in the far detector. The errors represent estimated shape uncertainties. 
TABLE VI. Observed IBD and estimated background rates per day for $1.2<E_{\mathrm{p}}<8 \mathrm{MeV}$.

\begin{tabular}{lcc}
\hline \hline Detector & Near & Far \\
\hline $\begin{array}{l}\text { IBD rate after } \\
\quad \text { background subtraction }\end{array}$ & $616.67 \pm 1.44$ & $61.24 \pm 0.42$ \\
Total background rate & $17.54 \pm 0.83$ & $3.14 \pm 0.23$ \\
DAQ live time (days) & 458.49 & 489.93 \\
Accidental rate & $6.89 \pm 0.09$ & $0.97 \pm 0.03$ \\
${ }^{9} \mathrm{Li} /{ }^{8} \mathrm{He}$ rate & $8.36 \pm 0.82$ & $1.54 \pm 0.23$ \\
Fast neutron rate & $2.28 \pm 0.04$ & $0.48 \pm 0.02$ \\
${ }^{252} \mathrm{Cf}$ contamination rate & $0.000 \pm 0.001$ & $0.14 \pm 0.03$ \\
\hline \hline
\end{tabular}

for the far detector. The energy-bin-uncorrelated spectral uncertainty is obtained from the measured background spectral error and is estimated as 0.025 events per day for the far detector. The energy-bin-correlated uncertainty is obtained from the fit error of the background rate in the region of $E_{\mathrm{p}}>8 \mathrm{MeV}$ and estimated as 0.015 events per day for the far detector.

\section{E. Summary of the backgrounds and their uncertainties}

The total remaining background rates for $1.2<E_{\mathrm{p}}<$ $8 \mathrm{MeV}$ in the final IBD candidate samples are estimated as $17.54 \pm 0.83$ (near) and $3.14 \pm 0.23$ (far) events per day. After the background subtraction, the IBD signal rates are $616.67 \pm 1.44$ (near) and $61.24 \pm 0.42$ (far) events per day. Table VI summarizes the observed IBD and estimated background rates. The live time is calculated as the sum of the duration of each physics data-taking run used in the analysis, and its uncertainty is estimated to be negligible.

Figures 23 and 24 show bin-to-bin uncorrelated and correlated uncertainties of measured background spectra, respectively. The largest uncertainty comes from the

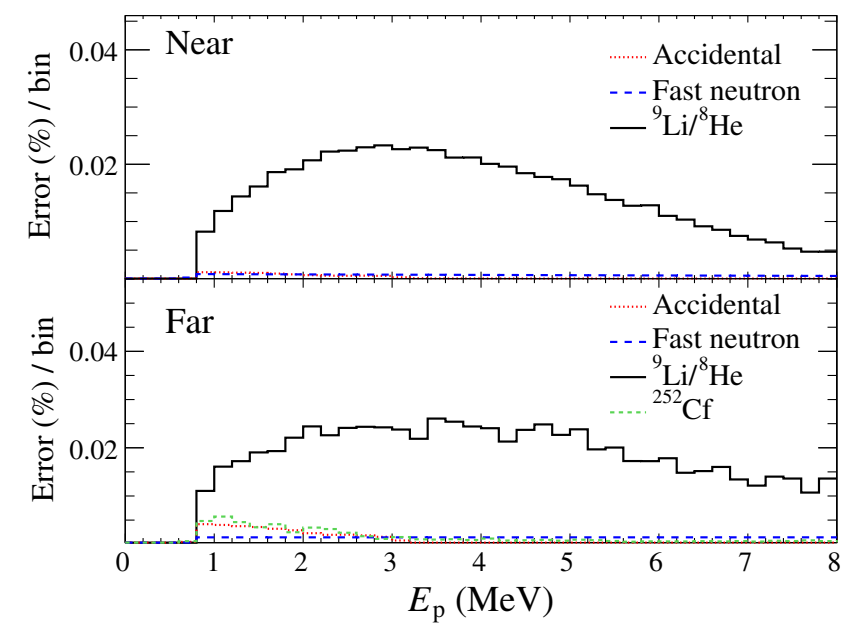

FIG. 23. Bin-to-bin uncorrelated spectral uncertainties of remaining backgrounds in the final IBD candidate samples. The ${ }^{9} \mathrm{Li} /{ }^{8} \mathrm{He}$ background is the most dominant source of the background uncertainty.

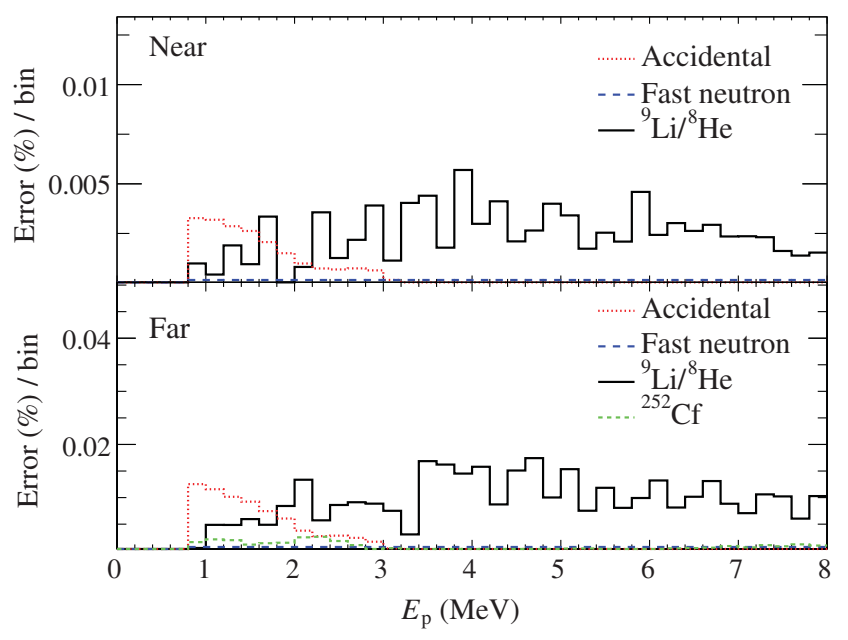

FIG. 24. Bin-to-bin correlated spectral uncertainties of remaining backgrounds in the final IBD candidate samples.

${ }^{9} \mathrm{Li} /{ }^{8} \mathrm{He}$ background. Note that the largest bin-to-bin correlated uncertainty at $E_{\mathrm{p}}<2.0 \mathrm{MeV}$ is due to the accidental background.

\section{EXPECTED REACTOR NEUTRINO FLUX}

The expected rates and spectra of reactor antineutrinos are calculated for the duration of physics data taking by taking into account the varying thermal powers, fission fractions of four fuel isotopes, energy release per fission, and fission and capture cross sections. The expected number of reactor $\bar{\nu}_{e}$ in a detector is computed using the following formula [23]:

$$
\begin{aligned}
n_{\nu} & =\frac{N_{\mathrm{p}}}{4 \pi L^{2}} \frac{\left[\sum_{i} \alpha_{i} \bar{\sigma}_{i}\right]}{\left[\sum_{i} \alpha_{i} E_{i}\right]} P_{\mathrm{th}} \\
& =\frac{N_{\mathrm{p}}}{4 \pi L^{2}} \frac{\sigma_{5}\left[1+\sum_{i} \alpha_{i}\left(\bar{\sigma}_{i} / \sigma_{5}-1\right)\right]}{E_{5}\left[1+\sum_{i} \alpha_{i}\left(E_{i} / E_{5}-1\right)\right]} P_{\mathrm{th}},
\end{aligned}
$$

where $N_{\mathrm{p}}$ is the number of free protons in target, $L$ is the distance between a reactor and a detector, $P_{\text {th }}$ is a reactor thermal power, $i$ is an index for each isotope of ${ }^{235} \mathrm{U},{ }^{238} \mathrm{U}$, ${ }^{239} \mathrm{Pu}$, and ${ }^{241} \mathrm{Pu}, \alpha_{i}$ is the fission fraction of the $i$ th isotope, $E_{i}\left(E_{5}\right)$ is the energy released by the $i$ th isotope $\left({ }^{235} \mathrm{U}\right), \bar{\sigma}_{i}=$ $\int \sigma\left(E_{\nu}\right) \phi_{i}\left(E_{\nu}\right) d E_{\nu}$ is the average fission cross section of the $i$ th isotope, and $\sigma_{5}$ is the cross section for ${ }^{235} \mathrm{U}$. Note that $\phi_{i}\left(E_{\nu}\right)$ is a $\bar{\nu}_{e}$ reference energy spectrum per isotope.

The average relative fission fractions of ${ }^{235} \mathrm{U},{ }^{238} \mathrm{U},{ }^{239} \mathrm{Pu}$, and ${ }^{241} \mathrm{Pu}$ during the $\sim 500$ live days of the data-taking period are $0.569: 0.073: 0.302: 0.056$ for the near detector and $0.572: 0.073: 0.299: 0.056$ for the far detector. These values are obtained by taking the weighted average of reactor cycles with reactor $\bar{\nu}_{e}$ fluxes according to the thermal outputs and baselines.

The thermal energy release per fission is given in Ref. [24], and its uncertainty introduces $0.2 \%$ for a correlated uncertainty. The daily thermal output measurement with a $0.5 \%$ 
uncertainty per reactor is provided by the Hanbit power plant [25]. The uncertainty is partially correlated between reactors. However, the uncertainty is weakly correlated between the near and far detectors because of multiple reactors and thus unknown information on an individual reactor $\bar{\nu}_{e}$ source.

The relative fission fraction of the four main isotopes are estimated with quoted $4 \%-10 \%$ uncertainties by the Hanbit power plant, using the ANC reactor simulation code [26]. The fission fraction uncertainties are consistent with other evaluations [27]. The resultant uncertainty in the expected reactor $\bar{\nu}_{e}$ flux is estimated as $0.7 \%$ using pseudoexperiments in which the relative isotope fractions are varied within their uncertainties. The fission fraction uncertainties for this analysis are assumed to be uncorrelated from reactor to reactor and from cycle to cycle although a large fraction of the uncertainty could be correlated among reactors according to Ref. [27]. Thus the uncertainty uncorrelated from reactor to reactor may be reduced in future work if the multireactor flux average is carefully treated. In the current analysis we have not attempted to reduce it because the energy-dependent variation due to the isotope fission fraction uncertainties is much smaller than the detector energy scale uncertainty. Based on the obtained thermal output and the relative fission fraction, an expected number of reactor $\bar{\nu}_{e}$ is obtained from Eq. (5) that can be rewritten as $n_{\nu}=\gamma_{0}(1+k(t)) P_{\text {th }}$. Note that $\gamma_{0}$ is determined by the experimental setup parameters and is a constant in time, and $k(t)$ is a time variation parameter of fuel isotopes. An expected number of reactor $\bar{\nu}_{e}$ events in a detector is calculated by adding all reactor contributions with individual baseline consideration and by taking into account cross section, live time, and detection efficiency.

The systematic uncertainties associated with the reactors are listed in Table VII. The reactor $\bar{\nu}_{e}$ flux uncertainties uncorrelated among reactors come from baseline distance, reactor thermal power, and fission fraction. The positions of two detectors and six reactors are surveyed with GPS and total station to determine the baseline distances between the detectors and reactors to an accuracy better than $10 \mathrm{~cm}$. Reactor $\bar{\nu}_{e}$ fluxes at the two detectors are obtained by calculating the flux reduction due to baseline distance to a precision better than $0.1 \%$. The baseline distance uncertainty is much smaller than the other two. The total

TABLE VII. Uncorrelated and correlated systematic uncertainties among reactors that are used in the reactor $\bar{\nu}_{e}$ flux estimation.

\begin{tabular}{lcc}
\hline \hline & Uncorrelated [\%] Correlated [\%] \\
\hline Baseline & 0.03 & $\ldots$ \\
Thermal power & 0.5 & $\ldots$ \\
Fission fraction & 0.7 & $\ldots$ \\
Fission reaction cross section & $\ldots$ & 0.5 \\
Reference energy spectra & $\ldots$ & 0.2 \\
Energy per fission & $\ldots$ & 2.0 \\
Combined & 0.9 & \\
\hline \hline
\end{tabular}

TABLE VIII. Expected number of reactor $\bar{\nu}_{e}$ in near and far detectors from each reactor without oscillation.

\begin{tabular}{lrc}
\hline \hline Reactor & Near & Far \\
\hline 1 & $4,1267(7.6 \%)$ & $7,860(15.1 \%)$ \\
2 & $90,463(16.6 \%)$ & $8,965(17.2 \%)$ \\
3 & $170,679(31.3 \%)$ & $8,657(16.6 \%)$ \\
4 & $155,431(28.5 \%)$ & $9,977(19.2 \%)$ \\
5 & $56,631(10.4 \%)$ & $8,362(16.1 \%)$ \\
6 & $30,132(5.5 \%)$ & $8,257(15.9 \%)$ \\
\hline \hline
\end{tabular}

uncorrelated uncertainty of reactor flux is estimated as $0.9 \%$. The correlated uncertainty in the fission reaction cross sections is found in Ref. [28], and the correlated uncertainty of reference energy spectra is given in Refs. [29,30]. The total correlated uncertainty is $2.0 \%$ and is canceled out in the far-to-near ratio measurement.

Table VIII shows an expected number of reactor $\bar{\nu}_{e}$ in near and far detectors from each reactor without oscillation.

\section{EXPECTED AND OBSERVED IBD RATES AND SPECTRA}

Figure 25 shows the measured daily rates of IBD candidates after background subtraction in the near and far detectors. The reactors were turned off for fuel replacement and maintenance. The expected rates assuming no oscillations are shown for comparison. The measured IBD rate in the far detector is clearly lower than the expected one, indicating the reactor $\bar{\nu}_{e}$ disappearance. The expected rates with the best-fit parameters are also shown and agree well with the measured IBD rates.

Figure 26 shows a spectral shape comparison between the observed IBD prompt spectrum after background subtraction and the prediction from a reactor $\bar{\nu}_{e}$ model $[29,30]$ using the far-to-near ratio measurement result. The fractional difference between the data and the prediction

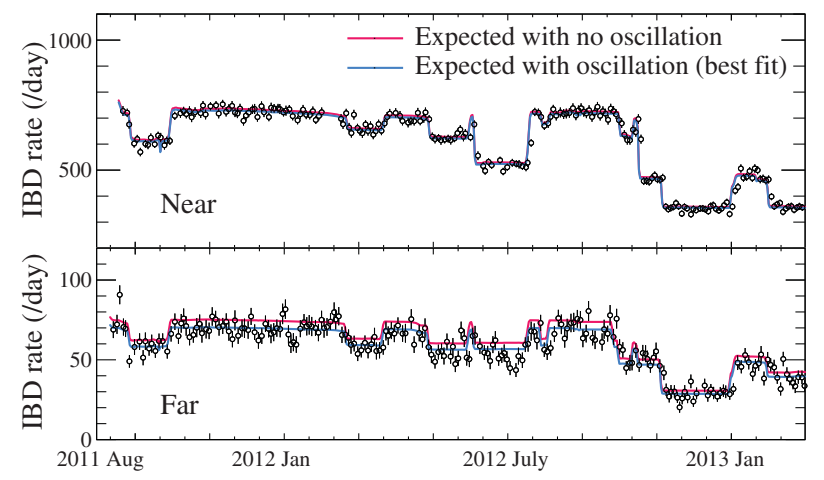

FIG. 25. Measured daily-average rates of reactor $\bar{\nu}_{e}$ after background subtraction in the near and far detectors as a function of running time. The red curves are the predicted rates for no oscillation. The blue curves are the predicted rates with the bestfit parameters and agree well with the measured one. 

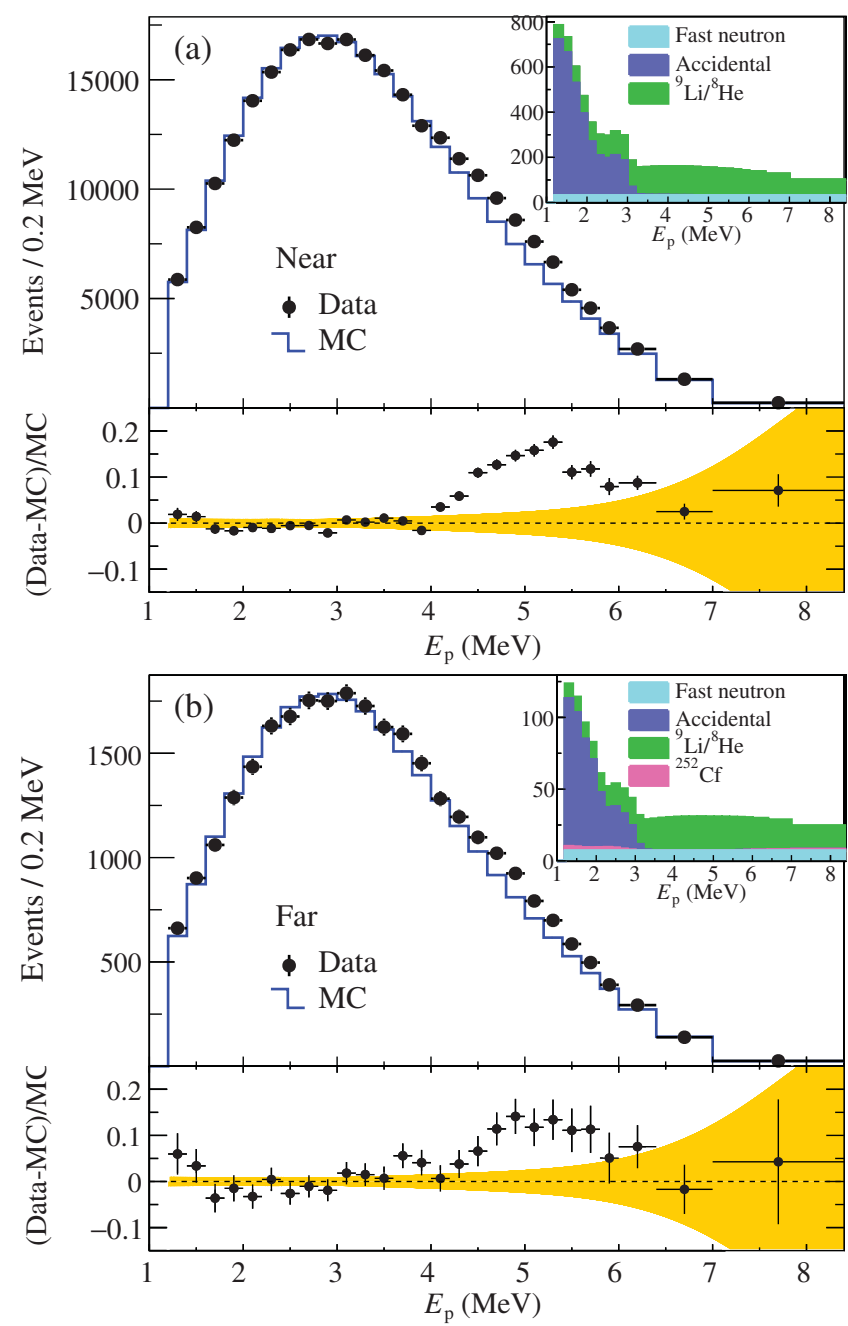

FIG. 26. Spectral shape comparison of observed and expected IBD prompt events in the (a) near and (b) far detectors. The observed spectra are obtained from subtracting the remaining background spectra as shown in the insets. The expected distributions are obtained from the best-fit oscillation results discussed later that are applied to the no-oscillation MC spectra. The expected spectra are normalized to data spectra in the region excluding $3.6<E_{\mathrm{p}}<6.6 \mathrm{MeV}$. The discrepancy between the data and MC prediction is clearly seen at $4-6 \mathrm{MeV}$. The observed excess is correlated with the reactor power and corresponds to $3 \%$ of the total number of IBD events. The deviation from the expectation is larger than the uncertainty of the expected spectrum (shaded band).

is also shown in the lower panel. A clear discrepancy is observed in the region of $5 \mathrm{MeV}$ in both detectors. To compare the spectral shape, the MC simulation predicted spectrum is normalized to the observed one in the region excluding $3.6<E_{\mathrm{p}}<6.6 \mathrm{MeV}$. The excess of events is estimated as about $3 \%$ of the total observed IBD events in both detectors. Furthermore, the $5 \mathrm{MeV}$ excess is observed to be proportional to the reactor thermal power where the rate is calculated from the events in excess at $3.6<E_{\mathrm{p}}<$ 6.6 $\mathrm{MeV}$ relative to the nominal model prediction $[29,30]$.

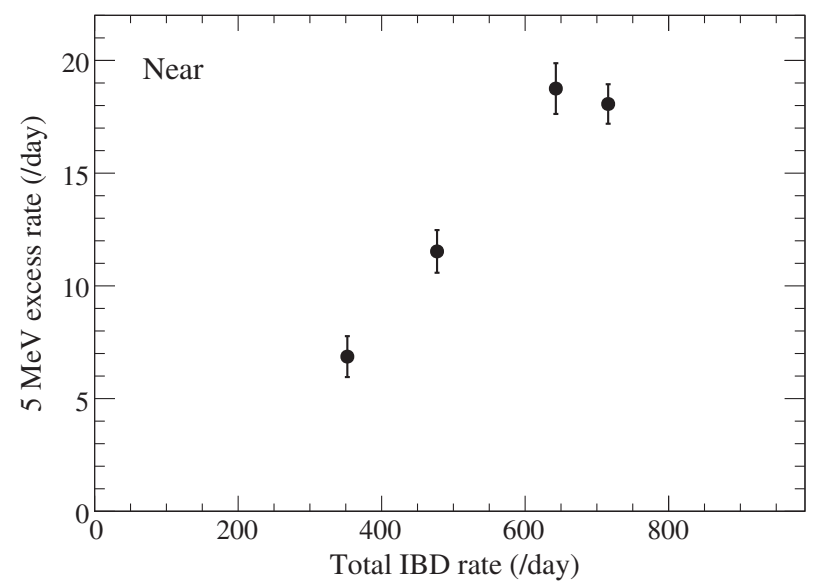

FIG. 27. Correlation between the $5 \mathrm{MeV}$ excess rate and the total IBD event rate in a total of 1400 live days of the near detector data. The error bar represents statistical uncertainty only.

Figure 27 shows a clear correlation between the $5 \mathrm{MeV}$ excess rate and the total IBD rate that corresponds to the reactor thermal power. This observation indicates that this excess indeed arises from the reactor $\bar{\nu}_{e}$ and thus suggests needs for reevaluation and modification of the current reactor $\bar{\nu}_{e}$ model $[29,30]$.

\section{SYSTEMATIC UNCERTAINTIES}

Systematic uncertainties of energy scale, backgrounds, detection efficiency, and reactor $\bar{\nu}_{e}$ flux are described in the earlier sections and summarized in Table IX. For the far-tonear ratio measurement the only uncorrelated systematic uncertainties contribute to the uncertainties of measured values. The energy-dependent detection efficiency is not considered in this analysis. Because of the difference in

TABLE IX. Summary of the systematic uncertainties. The detection efficiency uncertainty includes the systematic uncertainty for the signal loss due to the timing veto criteria given in Table V.

\begin{tabular}{lcc}
\hline \hline Uncertainty source & & Uncorrelated \\
\hline Reactor & & $0.9 \%$ \\
Detection efficiency & & $0.2 \%$ \\
+ timing veto & & $0.15 \%$ \\
Energy scale & & Bin-uncorrelated \\
& & $3.48 \%$ (near) \\
Total background & $3.18 \%$ (near) & $2.51 \%$ (far) \\
& $6.97 \%$ (far) & $0.30 \%$ (near) \\
Accidental & $1.19 \%$ (near) & $0.82 \%$ (far) \\
& $2.98 \%$ (far) & $1.01 \%$ (near) \\
Fast neutron & $1.45 \%$ (near) & $1.04 \%$ (far) \\
& $3.11 \%$ (far) & $7.28 \%$ (near) \\
${ }^{9} \mathrm{Li} /{ }^{8} \mathrm{He}$ & $6.58 \%$ (near) & $4.80 \%$ (far) \\
& $14.0 \%$ (far) & $20.8 \%$ (far) \\
${ }^{252} \mathrm{Cf}$ & $17.4 \%$ (far) & \\
\hline \hline
\end{tabular}


overburden, we assume no correlated uncertainties between the near and far detectors. Therefore, to be conservative, the background uncertainty of each detector is fully taken as an uncorrelated systematic one in a $\chi^{2}$ fit described later.

In summary, the relative energy-scale difference is $0.15 \%$, the uncorrelated systematic uncertainty of the detection efficiency is $0.2 \%$, the systematic uncertainties of the total backgrounds are $4.7 \%$ (near) and $7.3 \%$ (far), and the uncorrelated systematic uncertainty of reactor $\bar{\nu}_{e}$ flux is $0.9 \%$.

\section{RESULTS}

The relative measurement makes the method insensitive to correlated uncertainties between the near and far detectors and reduces uncorrelated reactor uncertainties. The measurement results are presented based on three different analysis methods to validate their consistencies. They are rate-only, rate and spectrum, and spectrum-only analyses. The results shown here are found in Ref. [11].

\section{A. Rate-only results}

In the rate-only analysis the oscillation amplitude of neutrino survival probability is extracted from the information on the observed reactor $\bar{\nu}_{e}$ rates only, without using the prompt energy spectra. We observe a clear deficit of reactor $\bar{\nu}_{e}$ in the far detector. Using the deficit information, a rate-only analysis obtains the value of $\sin ^{2} 2 \theta_{13}$ as $0.087 \pm 0.009$ (stat.) \pm 0.007 (syst.), where the world average value of $\left|\Delta m_{e e}^{2}\right|=(2.49 \pm 0.06) \times 10^{-3} \mathrm{eV}^{2}$ is used [31]. The $\chi^{2}$ fit for the result is described in Ref. [4]. The systematic error of $\sin ^{2} 2 \theta_{13}$ is reduced from 0.019 to 0.007 , mainly due to the reduced background rate and uncertainty, relative to the first measurement in 2012 [4]. In addition, the statistical error is reduced from 0.013 to 0.009 . Note that the largest reduction of the background rate and uncertainty comes from the ${ }^{9} \mathrm{Li} /{ }^{8} \mathrm{He}$ background.

\section{B. Rate and spectrum results}

In the rate and spectrum analysis the oscillation amplitude and frequency of neutrino survival probability are measured based on the information on the observed reactor $\bar{\nu}_{e}$ rates and spectra. We observe a clear energy-dependent deficit of reactor $\bar{\nu}_{e}$ in the far detector. Even with the unexpected structure around $5 \mathrm{MeV}$, the oscillation amplitude and frequency can be determined from a fit to the measured far-to-near ratio of IBD prompt spectra. The determination is not affected by the presence of the $5 \mathrm{MeV}$ excess because of its cancellation in the ratio measurement. For the determination of $\left|\Delta m_{e e}^{2}\right|$ and $\sin ^{2} 2 \theta_{13}$, a $\chi^{2}$ with pull parameter terms of systematic uncertainties is constructed using the spectral ratio measurement and is minimized by varying the oscillation parameters and pull parameters [32]. The following $\chi^{2}$ function is used for the rate and shape analysis:

$$
\begin{aligned}
\chi^{2}= & \sum_{i=1}^{\mathrm{N}_{\text {bins }}} \frac{\left(O_{i}^{\mathrm{F} / \mathrm{N}}-T_{i}^{\mathrm{F} / \mathrm{N}}\right)^{2}}{U_{i}^{\mathrm{F} / \mathrm{N}}}+\sum_{d=\mathrm{N}, \mathrm{F}}\left(\frac{b^{d}}{\sigma_{\text {bkg }}^{d}}\right)^{2} \\
& +\sum_{r=1}^{6}\left(\frac{f_{r}}{\sigma_{\text {flux }}^{r}}\right)^{2}+\left(\frac{\epsilon}{\sigma_{\text {eff }}}\right)^{2}+\left(\frac{\eta}{\sigma_{\text {scale }}}\right)^{2},
\end{aligned}
$$

where $O_{i}^{\mathrm{F} / \mathrm{N}}$ is the observed far-to-near ratio of IBD candidates in the $i$ th $E_{\mathrm{p}}$ bin after background subtraction, $T_{i}^{\mathrm{F} / \mathrm{N}}=T_{i}^{\mathrm{F} / \mathrm{N}}\left(b^{d}, f_{r}, \epsilon, \eta ; \theta_{13},\left|\Delta m_{e e}^{2}\right|\right) \quad$ is the expected far-to-near ratio of IBD events, and $U_{i}^{\mathrm{F} / \mathrm{N}}$ is the statistical uncertainty of $O_{i}^{\mathrm{F} / \mathrm{N}}$.

The expected ratio $T_{i}^{\mathrm{F} / \mathrm{N}}$ is calculated using the reactor $\bar{\nu}_{e}$ model, the IBD cross section, and the detection efficiency together with the signal loss due to the timing veto criteria, and folding the $\bar{\nu}_{e}$ survival probability and detector effects. The systematic uncertainty sources are embedded by pull parameters $\left(b^{d}, f_{r}, \epsilon\right.$, and $\left.\eta\right)$ with associated uncertainties $\left(\sigma_{\mathrm{bkg}}^{d}, \sigma_{\text {flux }}^{r}, \sigma_{\mathrm{eff}}\right.$, and $\left.\sigma_{\text {scale }}\right)$. The pull parameters allow variations from the expected far-to-near ratio of IBD events within their corresponding systematic uncertainties. The pull parameters $b^{d}$ and $\eta$ introduce deviations from the expected spectra accounting for the effects of the associated energy dependent systematic uncertainties. For the spectral deviations the energy-bin correlated and uncorrelated uncertainties are separately taken into account. The uncorrelated reactor-flux systematic uncertainty $\sigma_{\text {flux }}^{r}$ is $0.9 \%$, the uncorrelated detection and timing veto systematic uncertainty $\sigma_{\text {eff }}$ is $0.2 \%$, the uncorrelated energy-scale systematic uncertainty $\sigma_{\text {scale }}$ is $0.15 \%$, and the background uncertainty $\sigma_{\mathrm{bkg}}^{d}$ is $4.7 \%$ and $7.3 \%$ for near and far detectors, respectively. The $\chi^{2}$ is constructed as a sum of two periods, before ( $\sim 400$ days) and after ( $\sim 100$ days) ${ }^{252} \mathrm{Cf}$ contamination. A profile likelihood method is used to incorporate the systematic uncertainties in the fit. The best-fit values obtained from the rate and spectrum analysis are $\sin ^{2} 2 \theta_{13}=0.082 \pm 0.009$ (stat.) \pm 0.006 (syst.) and $\left|\Delta m_{e e}^{2}\right|=$ $\left[2.62_{-0.23}^{+0.21} \text { (stat. }\right)_{-0.13}^{+0.12}$ (syst.) $] \times 10^{-3} \mathrm{eV}^{2}$ with $\chi^{2} / \mathrm{NDF}=$ $58.9 / 66$, where NDF is the number of degrees of freedom. This result is consistent with that of the rate-only analysis within their errors. Another fit result is also obtained assuming an independent pull parameter for each energy bin to allow maximum variation of the background shapes within their uncertainties. The total systematic errors for both $\sin ^{2} 2 \theta_{13}$ and $\left|\Delta m_{e e}^{2}\right|$ remain almost unchanged by the fit.

Table X presents systematic uncertainties of $\sin ^{2} 2 \theta_{13}$ and $\left|\Delta m_{e e}^{2}\right|$ from several uncertainty sources. The uncertainties of energy-scale and backgrounds are the dominant sources of the total systematic uncertainty for $\left|\Delta m_{e e}^{2}\right|$. The measured value of $\left|\Delta m_{e e}^{2}\right|$ corresponds to $\left|\Delta m_{31}^{2}\right|=\left(2.64_{-0.26}^{+0.24}\right) \times$ $10^{-3} \mathrm{eV}^{2} \quad\left(\left|\Delta m_{31}^{2}\right|=\left[2.60_{-0.26}^{+0.24}\right] \times 10^{-3} \mathrm{eV}^{2}\right)$ for the normal (inverted) neutrino mass ordering, using measured 
TABLE X. Systematic uncertainties from various uncertainty sources. The dominant sources of the total systematic uncertainties for $\left|\Delta m_{e e}^{2}\right|$ are the uncertainties of energy scale and backgrounds.

\begin{tabular}{lcc}
\hline \hline & $\delta\left|\Delta m_{e e}^{2}\right|\left(\times 10^{-3} \mathrm{eV}^{2}\right)$ & $\delta\left(\sin ^{2} 2 \theta_{13}\right)$ \\
\hline Reactor & $+0.018,-0.018$ & $+0.0026,-0.0028$ \\
Detection efficiency & $+0.020,-0.022$ & $+0.0028,-0.0029$ \\
Energy scale & $+0.081,-0.094$ & $+0.0026,-0.0015$ \\
Backgrounds & $+0.084,-0.106$ & $+0.0030,-0.0028$ \\
Total & $+0.115,-0.133$ & $+0.0055,-0.0052$ \\
\hline \hline
\end{tabular}

oscillation parameters of $\sin ^{2} 2 \theta_{12}=0.846 \pm 0.021$ and $\Delta m_{21}^{2}=(7.53 \pm 0.18) \times 10^{-3} \mathrm{eV}^{2}[31]$.

Figure 28 shows the background-subtracted, observed spectrum at the far detector compared to the one expected with no oscillation and the one expected with the best-fit oscillation parameters at the far detector. The expected spectrum with no oscillation is obtained by weighting the spectrum at the near detector with no-oscillation assumption in order to include the $5 \mathrm{MeV}$ excess. The expected spectrum with the best-fit oscillation parameters is obtained by applying the measured values of $\sin ^{2} 2 \theta_{13}$ and $\left|\Delta m_{e e}^{2}\right|$ to the one expected with no oscillation at the far

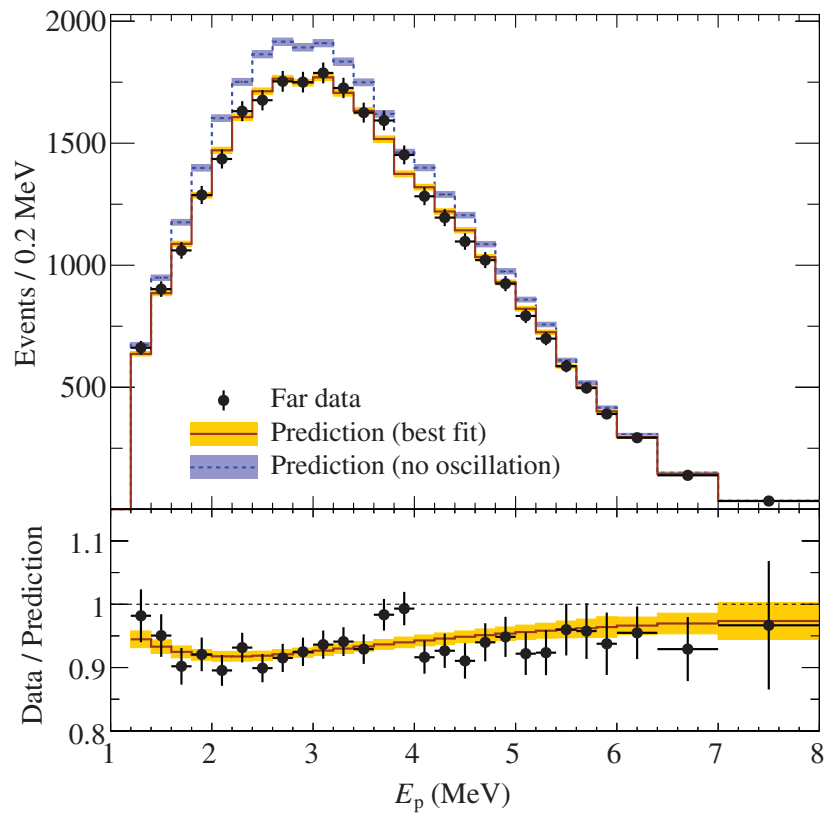

FIG. 28. Top: Comparison of the observed IBD prompt spectrum in the far detector (dots) with the no-oscillation prediction (blue shaded histogram) obtained from the measurement in the near detector. The prediction from the best-fit oscillation parameters is also shown (yellow shaded histogram). Both blue and yellow bands represent uncertainties. Bottom: Ratio of IBD events measured in the far detector to the nooscillation prediction (dots) and the ratio from the MC simulation with best-fit results folded in (shaded band). Errors are statistical uncertainties only, although both statistical and systematic uncertainties are included in the $\chi^{2}$ fitting. detector. The observed spectrum at the far detector shows a clear energy dependent disappearance of reactor $\bar{\nu}_{e}$ events consistent with neutrino oscillations. A weak deviation from the expectation is observed near $E_{\mathrm{p}}=3.8 \mathrm{MeV}$ in Fig. 28 and will be kept monitored for its persistency with more data. Figure 29 shows $68.3 \%, 95.5 \%$, and $99.7 \%$ C.L. allowed regions for the neutrino oscillation parameters $\left|\Delta m_{e e}^{2}\right|$ and $\sin ^{2} 2 \theta_{13}$. The results from other reactor experiments $[33,34]$ are also shown in the figure.

\section{Spectrum-only results}

The spectrum-only analysis uses only spectral shape information with a free normalization that allows variation in the expected IBD signal rates. This method obtains the oscillation frequency of $\left|\Delta m_{e e}^{2}\right|$ from the energy dependent disappearance of the reactor $\bar{\nu}_{e}$ without using the information on the total-rate deficit, although it does not provide a sensitive measurement of $\sin ^{2} 2 \theta_{13}$. The spectrumonly analysis yields $\left|\Delta m_{e e}^{2}\right|=\left(2.62_{-0.41}^{+0.38}\right) \times 10^{-3} \mathrm{eV}^{2}$ and $\sin ^{2} 2 \theta_{13}=0.066_{-0.046}^{+0.042}$ with $\chi^{2} / \mathrm{NDF}=58.8 / 67$. This result is consistent with those from the rate and spectrum analysis and the rate-only analysis within the errors.

\section{Energy and baseline dependent reactor $\bar{\nu}_{e}$ disappearance}

The survival probability of reactor $\bar{\nu}_{e}$ is a function of a baseline $L$ over neutrino energy $E_{\nu}$ as written in Eq. (1). Because of having multiple reactors as neutrino sources, an effective baseline $L_{\text {eff }}$ is defined by the reactor-detector distance weighted by the IBD event rate from each reactor. Note that $L_{\text {eff }}$ is time dependent due to the IBD event rate weighting. The neutrino energy $E_{\nu}$ is converted from the

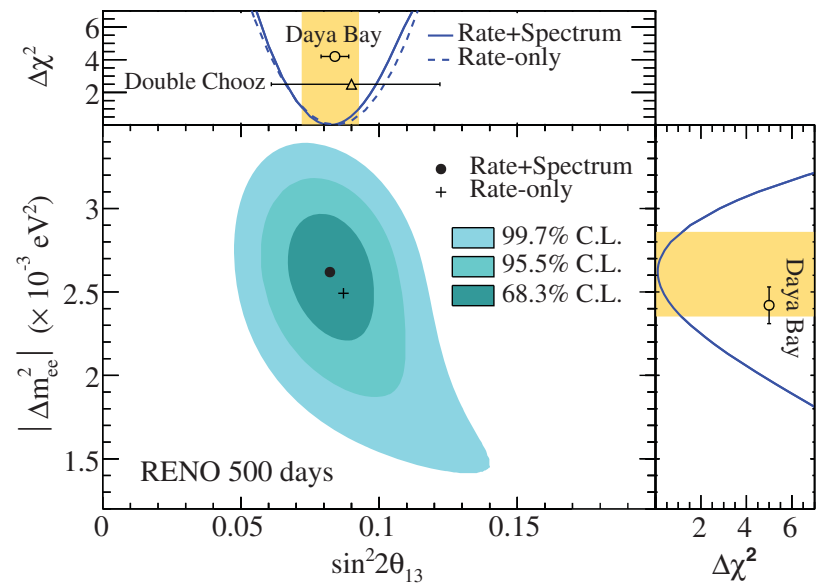

FIG. 29. Allowed regions of $68.3 \%, 95.5 \%$, and 99.7\% C.L. in the $\left|\Delta m_{e e}^{2}\right|$ vs $\sin ^{2} 2 \theta_{13}$ plane. The best-fit values are shown as the black dot. The $\Delta \chi^{2}$ distribution for $\sin ^{2} 2 \theta_{13}$ (top) and $\left|\Delta m_{e e}^{2}\right|$ (right) are also shown with a $1 \sigma$ band. The rate-only result for $\sin ^{2} 2 \theta_{13}$ is shown as the cross. The results from Daya Bay [33] and Double Chooz [34] Collaborations are also shown for comparison. 


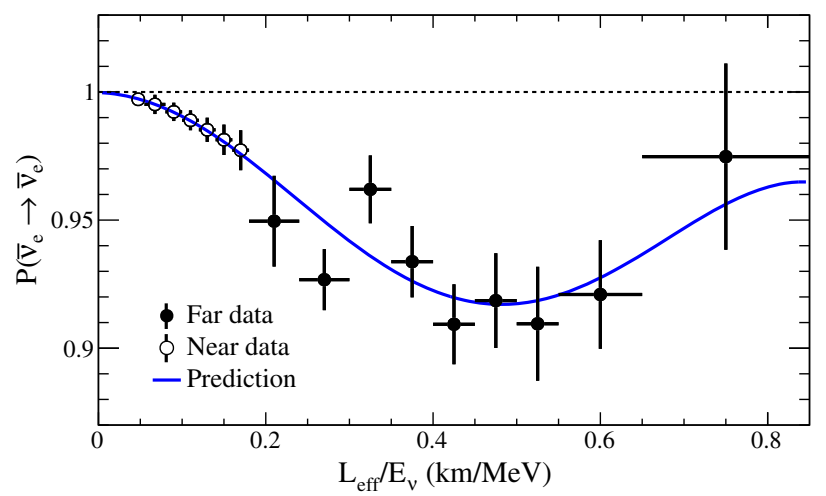

FIG. 30. Measured reactor $\bar{\nu}_{e}$ survival probability in the far detector as a function of $L_{\text {eff }} / E_{\nu}$. The curve is a predicted survival probability, obtained from the observed probability in the near detector, for the best-fit values of $\left|\Delta m_{e e}^{2}\right|$ and $\sin ^{2} 2 \theta_{13}$. The $L_{\text {eff }} / E_{\nu}$ value of each data point is given by the average of the counts in each bin.

IBD prompt energy. A daily $L_{\text {eff }} / E_{\nu}$ distribution of the IBD events is obtained from the background subtracted IBD event spectrum and the daily $L_{\text {eff. The }}$ observed $L_{\text {eff }} / E_{\nu}$ distribution is obtained by summing up the daily distributions weighted by a daily IBD rate. The measured survival probability is obtained by the ratio of the observed IBD events to the expected ones with no oscillation in each bin of $L_{\text {eff }} / E_{\nu}$. Figure 30 shows the measured survival probability of reactor $\bar{\nu}_{e}$ in the far detector as a function of $L_{\text {eff }} / E_{\nu}$. A predicted survival probability is obtained from the observed probability distribution in the near detector and the best-fit oscillation values. Because of the observed $5 \mathrm{MeV}$ excess, the expected $L_{\text {eff }} / E_{\nu}$ distribution is derived from the measured spectrum in the near detector instead of the IBD MC spectrum. A clear $L_{\text {eff }} / E_{\nu}$-dependent disappearance of reactor $\bar{\nu}_{e}$ is observed and demonstrates the periodic feature of neutrino oscillation.

\section{SUMMARY AND PROSPECTS}

Using about 500 live days of data RENO has observed a clear energy dependent disappearance of reactor $\bar{\nu}_{e}$ using two identical detectors and obtained $\sin ^{2} 2 \theta_{13}=0.082 \pm$ 0.010 and $\left|\Delta m_{e e}^{2}\right|=\left[2.62_{-0.26}^{+0.24}\right] \times 10^{-3} \mathrm{eV}^{2}$ based on the measured disappearance expected from neutrino oscillations. RENO has measured $\sin ^{2} 2 \theta_{13}$ more precisely and
TABLE XI. Comparison of the measured $\sin ^{2} 2 \theta_{13}$ and $\left|\Delta m_{e e}^{2}\right|$ between the first measurement in 2012 [4] and the current measurement.

\begin{tabular}{lcc}
\hline \hline Results & 2012 & Current \\
\hline Live days & 220 & 500 \\
$\sin ^{2} 2 \theta_{13}$ & $0.113 \pm 0.023$ & $0.082 \pm 0.011$ \\
Precision & $20.4 \%$ & $13.4 \%$ \\
$\left|\Delta m_{e e}^{2}\right|\left(\times 10^{-3} \mathrm{eV}^{2}\right)$ & $2.32($ PDG 2012) & $2.62 \pm 0.26$ \\
Precision & $\ldots$ & $9.9 \%$ \\
\hline \hline
\end{tabular}

$\left|\Delta m_{e e}^{2}\right|$ for the first time with the rate and spectrum analysis. The systematic uncertainty of $\sin ^{2} 2 \theta_{13}$ has been significantly reduced from 0.019 [4] to 0.006 due to the improvement in reducing the background uncertainties, especially the most dominant ${ }^{9} \mathrm{Li} /{ }^{8} \mathrm{He}$ background rate and its uncertainty. A clear IBD spectral difference from the current reactor $\bar{\nu}_{e}$ model is observed in the region of $5 \mathrm{MeV}$ in both detectors, with an excess corresponding to about $3 \%$ of the total observed IBD events. The observed excess is clearly correlated with the reactor thermal power, indicating the excess arises from the reactor $\bar{\nu}_{e}$.

Table XI presents a comparison of the measured values of $\sin ^{2} 2 \theta_{13}$ and $\left|\Delta m_{e e}^{2}\right|$ between the first RENO measurement in 2012 [4] and the current measurement. The precision on $\sin ^{2} 2 \theta_{13}$ is improved from $20.4 \%$ to $13.4 \%$, and the $\left|\Delta m_{e e}^{2}\right|$ precision is $9.9 \%$. The background systematic uncertainties estimated from data are expected to be reduced with more data. The precision is expected to be $\sim 5 \%$ for both oscillation parameters with ten live years of data.

\section{ACKNOWLEDGMENTS}

The RENO experiment is supported by the National Research Foundation of Korea (NRF) Grant No. 20090083526 funded by the Korea Ministry of Science and ICT. Some of us have been supported by a fund from the BK21 of NRF. We gratefully acknowledge the cooperation of the Hanbit Nuclear Power Site and the Korea Hydro \& Nuclear Power Co., Ltd. (KHNP). We thank KISTI for providing computing and network resources through GSDC, and all the technical and administrative people who greatly helped in making this experiment possible.
[1] Y. Fukuda et al. (Super-Kamiokande Collaboration), Phys. Rev. Lett. 81, 1562 (1998).

[2] Q. R. Ahmad et al. (SNO Collaboration), Phys. Rev. Lett. 87, 071301 (2001); 89, 011301 (2002).
[3] F. P. An et al. (Daya Bay Collaboration), Phys. Rev. Lett. 108, 171803 (2012).

[4] J. K. Ahn et al. (RENO Collaboration), Phys. Rev. Lett. 108, 191802 (2012). 
[5] B. Pontecorvo, Zh. Eksp. Teor. Fiz. 34, 247 (1957) [Sov. Phys. JETP 7, 172 (1958)].

[6] Z. Maki, M. Nakagawa, and S. Sakata, Prog. Theor. Phys. 28, 870 (1962).

[7] K. Abe et al. (T2K Collaboration), Phys. Rev. D 88, 032002 (2013); Phys. Rev. Lett. 112, 061802 (2014).

[8] J. Bian et al. (NOvA Collaboration), arXiv:hep-ex/ 1510.05708 .

[9] S. T. Petcov and M. Piai, Phys. Lett. B 533, 94 (2002).

[10] H. Nunokawa, S. Parke, and R. Z. Funchal, Phys. Rev. D 72, 013009 (2005); S. Parke, Phys. Rev. D 93, 053008 (2016).

[11] J. H. Choi et al. (RENO Collaboration), Phys. Rev. Lett. 116, 211801 (2016).

[12] K. S. Park et al. (RENO Collaboration), Nucl. Instrum. Methods Phys. Res., Sect. A 686, 91 (2012).

[13] K. J. Ma et al. (RENO Collaboration), Nucl. Instrum. Methods Phys. Res., Sect. A 629, 93 (2011).

[14] J. S. Park et al. (RENO Collaboration), Nucl. Instrum. Methods Phys. Res., Sect. A 707, 45 (2013).

[15] J. K. Ahn et al. (RENO Collaboration), arXiv:1003.1391.

[16] S. Yamada et al., IEEE Trans. Nucl. Sci. 57, 428 (2010); H. Nishino, K. Awai, Y. Hayato, S. Nakayama, K. Okumura, M. Shiozawa, A. Takeda, K. Ishikawa, A. Minegishi, and Y. Arai, Nucl. Instrum. Methods Phys. Res., Sect. A 610, 710 (2009); T. Uchida, IEEE Trans. Nucl. Sci. 55, 1631 (2008).

[17] J. H. Choi et al. (RENO Collaboration), Nucl. Instrum. Methods Phys. Res., Sect. A 810, 100 (2016).

[18] G. A. Horton-Smith, Generic liquid scintillator Genat4 simulation, http://neutrino.phys.ksu.edu/GLG4sim/, 2005.

[19] H. S. Kim et al. (RENO Collaboration), arXiv:1609.09483.

[20] H. S. Kim, Sae Mulli 62, 631 (2012).
[21] P. Vogel and J. Beacom, Phys. Rev. D 60, 053003 (1999).

[22] National Nuclear Data Center, Brookhaven National Laboratory, http://www.nndc.bnl.gov/sg21/nucl/indc-nds-440 .pdf (2013).

[23] Yu. V. Klimov, V. I. Kopeikin, L. A. Mikaélyan, K. V. Ozerov, and V. V. Sinev, Energy 76, 123 (1994).

[24] V. Kopeikin, L. A. Mikaelyan, and V. V. Sinev, Phys. At. Nucl. 67, 1892 (2004).

[25] S. F. E. Tournu et al., Improving Pressurized Water Reactor Performance Through Instrumentation: Application Case of Reducing Uncertainties on Thermal Power (EPRI, Palo Alto, CA, 2001), p. 1001470.

[26] ANC: A Westinghouse Advanced Nodal Computing Code, Westinghouse Report No. WCAP-10965-P-A(P), 1986.

[27] Z. Djurcic, J. A. Detwiler, A. Piepke, V. R. Foster, L. Miller, and G. Gratta, J. Phys. G 36, 045002 (2009); X. B. Ma, F. Lu, L. Z. Wang, Y. X. Chen, W. L. Zhong, and F. P. An, Mod. Phys. Lett. A 31, 1650120 (2016); X. B. Ma, R. M. Qiu, and Y. X. Chen, Nucl. Phys. A958, 211 (2017).

[28] G. Mention, M. Fechner, Th. Lasserre, Th. A. Mueller, D. Lhuillier, M. Cribier, and A. Letourneau, Phys. Rev. D 83, 073006 (2011).

[29] Th. A. Mueller et al., Phys. Rev. C 83, 054615 (2011).

[30] P. Huber, Phys. Rev. C 84, 024617 (2011); 85, 029901(E) (2012).

[31] K. A. Olive et al. (Particle Data Group Collaboration), Chin. Phys. C 38, 090001 (2014).

[32] K. Anderson et al., arXiv:hep-ex/0402041.

[33] F. P. An et al. (Daya Bay Collaboration), Phys. Rev. Lett. 112, 061801 (2014); 115, 111802 (2015).

[34] Y. Abe et al. (Double Chooz Collaboration), J. High Energy Phys. 10 (2014) 86. 